Journal of

Synchrotron

Radiation

ISSN 0909-0495

Editor: G. Ice

\title{
X-Treme beamline at SLS: X-ray magnetic circular and linear dichroism at high field and low temperature
}

\author{
Cinthia Piamonteze, Uwe Flechsig, Stefano Rusponi, Jan Dreiser, Jakoba \\ Heidler, Marcus Schmidt, Reto Wetter, Marco Calvi, Thomas Schmidt, \\ Helena Pruchova, Juraj Krempasky, Christoph Quitmann, Harald Brune \\ and Frithjof Nolting
}

J. Synchrotron Rad. (2012). 19, 661-674

Copyright (C) International Union of Crystallography

Author(s) of this paper may load this reprint on their own web site or institutional repository provided that this cover page is retained. Republication of this article or its storage in electronic databases other than as specified above is not permitted without prior permission in writing from the IUCr.

For further information see http://journals.iucr.org/services/authorrights.html

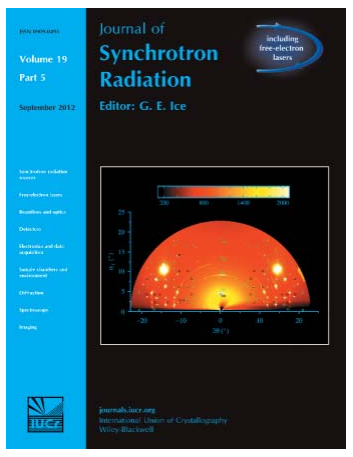

Synchrotron radiation research is rapidly expanding with many new sources of radiation being created globally. Synchrotron radiation plays a leading role in pure science and in emerging technologies. The Journal of Synchrotron Radiation provides comprehensive coverage of the entire field of synchrotron radiation research including instrumentation, theory, computing and scientific applications in areas such as biology, nanoscience and materials science. Rapid publication ensures an up-to-date information resource for scientists and engineers in the field.

Crystallography Journals Online is available from journals.iucr.org 
Journal of

Synchrotron

ISSN 0909-0495

Accepted 19 June 2012
Radiation

Received 6 February 2012

\section{X-Treme beamline at SLS: X-ray magnetic circular and linear dichroism at high field and low temperature}

\author{
Cinthia Piamonteze, ${ }^{\mathrm{a} *}$ Uwe Flechsig, ${ }^{\mathrm{a}}$ Stefano Rusponi, ${ }^{\mathrm{b}}$ Jan Dreiser, ${ }^{\mathrm{a}}$ \\ Jakoba Heidler, ${ }^{a}$ Marcus Schmidt, ${ }^{a}$ Reto Wetter, ${ }^{a}$ Marco Calvi, ${ }^{a}$ Thomas Schmidt, ${ }^{a}$ \\ Helena Pruchova, ${ }^{a}$ Juraj Krempasky, ${ }^{a}$ Christoph Quitmann, ${ }^{a}$ Harald Brune $^{b}$ and \\ Frithjof Nolting ${ }^{\mathrm{a}}$
}

\author{
${ }^{\mathbf{a}}$ Swiss Light Source, Paul Scherrer Institut, CH-5232 Villigen PSI, Switzerland, and ${ }^{\mathbf{b}}$ Institute of \\ Condensed Matter Physics (ICMP), École Polytechnique Fédérale de Lausanne (EPFL), CH-1015 \\ Lausanne, Switzerland. E-mail: cinthia.piamonteze@psi.ch
}

\begin{abstract}
$\mathrm{X}$-Treme is a soft X-ray beamline recently built in the Swiss Light Source at the Paul Scherrer Institut in collaboration with École Polytechnique Fédérale de Lausanne. The beamline is dedicated to polarization-dependent X-ray absorption spectroscopy at high magnetic fields and low temperature. The source is an elliptically polarizing undulator. The end-station has a superconducting $7 \mathrm{~T}-2 \mathrm{~T}$ vector magnet, with sample temperature down to $2 \mathrm{~K}$ and is equipped with an in situ sample preparation system for surface science. The beamline commissioning measurements, which show a resolving power of 8000 and a maximum flux at the sample of $4.7 \times 10^{12}$ photons s$^{-1}$, are presented. Scientific examples showing X-ray magnetic circular and X-ray magnetic linear dichroism measurements are also presented.
\end{abstract}

(C) 2012 International Union of Crystallography Printed in Singapore - all rights reserved
Keywords: X-ray absorption spectroscopy; X-ray magnetic circular dichroism; $X$-ray linear dichroism; soft $X$-rays; instrumentation.

\section{Introduction}

Polarization-dependent X-ray absorption spectroscopy (XAS) includes a number of techniques, the most common being: $\mathrm{X}$-ray magnetic circular dichroism (XMCD), X-ray magnetic linear dichroism (XMLD) and X-ray natural linear dichroism (XNLD). A large magneto-optical effect was predicted in the pioneering work of Erskine \& Stern (1975) for Ni $M_{2,3}$-edges based on calculations taking into account the large spin-orbit coupling of the $3 p$ states and the spin polarization of the $3 d$ states. Ten years later, Thole et al. (1985) predicted a strong dichroism in the $M_{4,5}$-edges of rare-earth ions. Shortly after, the first XMLD measurements were performed on lanthanides $M$-edges by the same group (van der Laan et al., 1986). The first XMCD spectrum was measured in the hard X-ray energy range (Schütz et al., 1987), followed a few years later by the first measurement in the soft X-ray range by Chen et al. (1990) on Ni bulk sample. Since its early times, XMCD has been widely used for the study of a variety of questions related to magnetism such as: magnetic anisotropy (Stöhr \& Konig, 1995; Stöhr, 1999) and exchange bias (Ohldag et al., 2003) at interfaces; element-specific magnetic properties of bimetallic paramagnetic molecules (Arrio et al., 1999); magnetic anisotropies, spin and orbital moments of single adatoms (Gambardella et al., 2003), of a submonolayer of single- molecule magnets (Mannini et al., 2009) or organometallic molecules on magnetic surfaces (Wäckerlin et al., 2010). $\mathrm{XMCD}$ has also been used in areas as wide as mineralogy (Pattrick et al., 2001), environmental sciences (Coker et al., 2006) and in biological systems such as metalloproteins (Funk et al., 2004, 2005). A few characteristics that make this technique unique are its element specificity, the direct access to the states responsible for the chemical bonding and magnetic properties as well as the possibility to extract quantitative information on the spin and orbital moment separately through sum rules (Thole et al., 1992; Carra et al., 1993). In addition, its high sensitivity is unreached by other techniques and enables the study of ultra-thin films and of magnetic impurities.

The X-Treme beamline operates in the soft X-ray energy range, from 400 to $1800 \mathrm{eV}$. This range includes, for example, the $L_{2,3^{-}}$edges of $3 d$ transition metals $(2 p \rightarrow 3 d)$, the $M_{2,3^{-}}$ edges from $4 d$ transition metals $(3 p \rightarrow 4 d)$, the $M_{4,5}$-edges from lanthanides $(3 d \rightarrow 4 f)$ and the $K$-edges of some common ligands such as nitrogen, oxygen and fluorine $(1 s \rightarrow 2 p)$. It is therefore the most interesting energy range for the study of the relevant states related to magnetism. The end-station contains a superconducting vector-magnet allowing an applied magnetic field of $7 \mathrm{~T}$ along the beam direction and of $2 \mathrm{~T}$ perpendicular to it in the horizontal plane. The superposition 
of both fields can be used to create a vector field up to $2 \mathrm{~T}$. The sample temperature can be adjusted from $370 \mathrm{~K}$ down to $2 \mathrm{~K}$. The beamline is installed in the Swiss Light Source (SLS) at the Paul Scherrer Institut and operated in collaboration with École Polytechnique Fédérale de Lausanne (EPFL). The SLS is a third-generation source with an electron storage ring energy of $2.4 \mathrm{GeV}$ and a current of $400 \mathrm{~mA}$, operated in top-up mode.

\section{Beamline specifications}

The X-Treme beamline is installed in the straight section $7 \mathrm{M}$ of SLS. The source is an Apple II undulator (Sasaki et al., 1993) with $54 \mathrm{~mm}$ period and total length of $1.7 \mathrm{~m}$. The polarization can be varied between circular right/left and linear from $0^{\circ}$ to $90^{\circ}$. The undulator operates in the energy range from $150 \mathrm{eV}$ up to $8000 \mathrm{eV}$, which corresponds to operation up to the 31 st harmonic. The source is shared alternatingly between two beamlines: X-Treme (400-1800 eV) and Phoenix $(800-8000 \mathrm{eV})$. The beam switch is made by inserting or removing the first
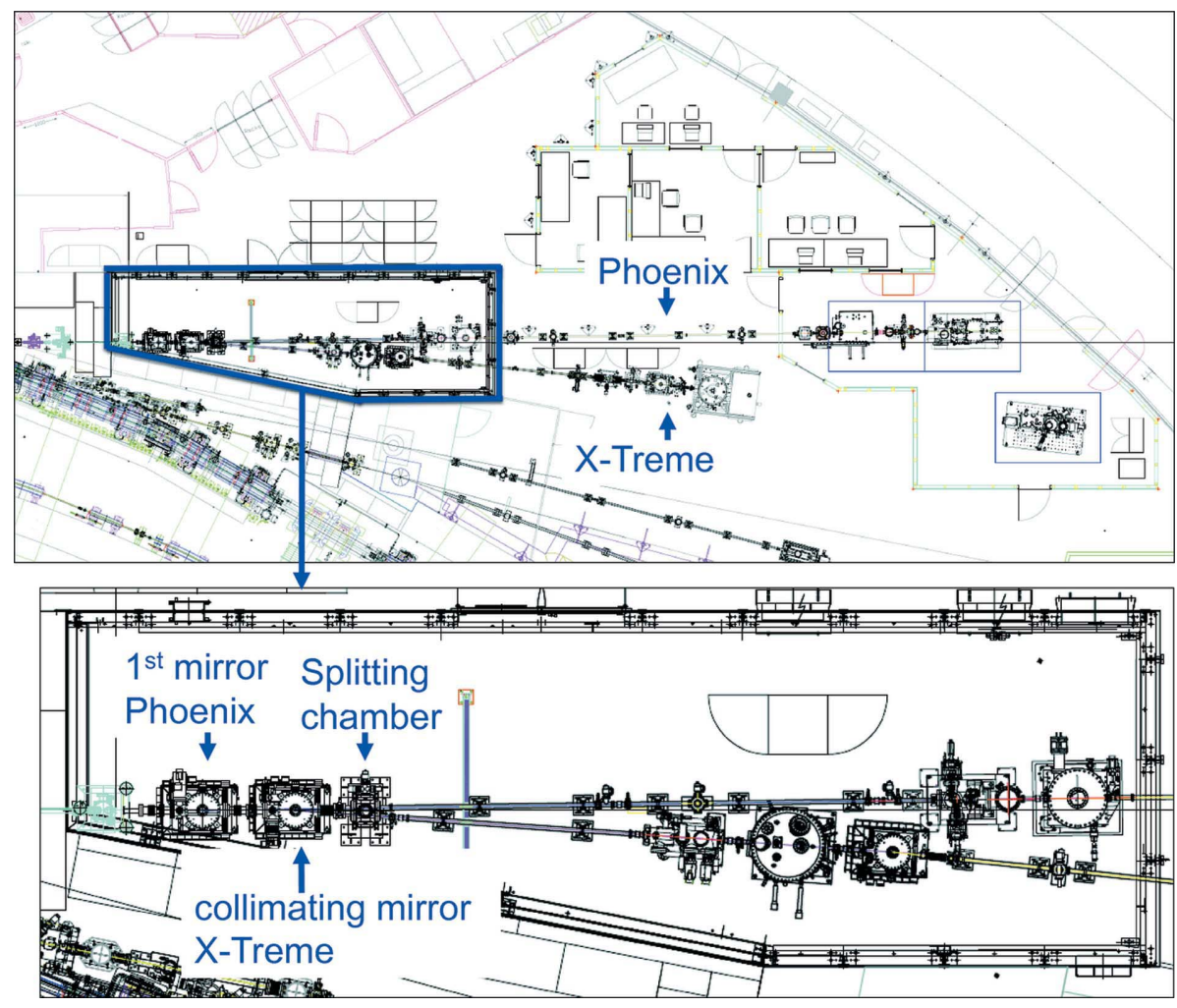

Figure 1

Floor layouts of the X-Treme and Phoenix beamlines. Top: overview of the two beamlines up to the experimental station. Bottom: zoom into the lead hutch. The first mirror from the Phoenix beamline and the collimating mirror from the X-Treme beamline are used for switching between the two branches. mirror of each beamline. Fig. 1 shows a top view of the two beamlines, with a zoom of the lead hutch where the mirrors used for the switching are marked. ${ }^{1}$ Downstream from the splitting chamber the vacuum system of the two beamlines is separated.

The beamline design is based on a plane-grating monochromator (PGM) operated with collimated light. ${ }^{2}$ In the PGM design the fix-focus constant $\left(c_{\mathrm{ff}}\right)$ is kept independent of the energy (Petersen, 1982). $c_{\mathrm{ff}}$ is given by

$$
c_{\mathrm{ff}}=\cos \beta / \cos \alpha,
$$

where $\alpha$ and $\beta$ are indicated in the schematic representation given in Fig. 2 and are defined in the grating equation,

$$
m \lambda / d=\sin \alpha+\sin \beta,
$$

where $m$ is the diffraction order, $\lambda$ is the photon wavelength and $d$ is the line spacing.

The deflection angle by the plane mirror $(\theta$, see Fig. 2$)$ is given by

$$
2 \theta=\alpha-\beta
$$

Therefore, the operation of the monochromator can be fully described by $\beta$ and $\theta$ and the grating equation is rewritten as

\footnotetext{
$\mathbf{1}$ The mirror chambers were manufactured by FMB Feinwerk \& Messtechnik, Berlin, Germany (http://www.fmb-berlin.de/).

2 The monochromator chamber was manufactured by Jenoptik, Jena, Germany (http://www.jenoptik.com/).
}

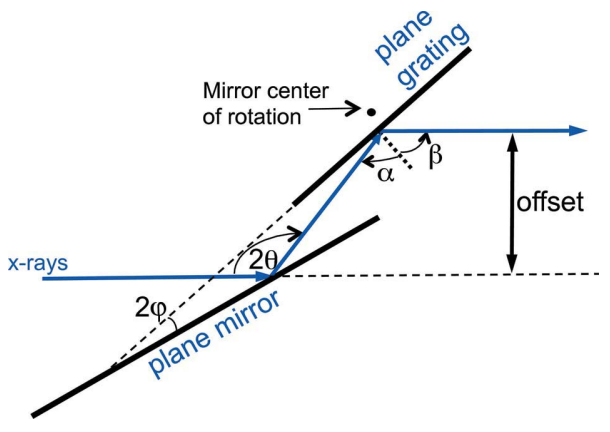

Figure 2

Scheme of the plane-grating monochromator indicating the relevant angles.

$$
m \lambda / d=2 \cos \theta \sin (\theta+\beta) .
$$

The X-Treme monochromator is operated in inside diffraction order $\left(m>0, c_{\mathrm{ff}}>1\right)$. The light accepted by the monochromator is previously collimated in the vertical direction by the first mirror. The PGM operation with collimated light allows the value of $c_{\mathrm{ff}}$ to freely vary (Follath \& Senf, 1997). This design gives an additional flexibility, since by changing the $c_{\mathrm{ff}}$ value the monochromator can be optimized for flux, energy resolution or harmonic rejection as described by Follath (2001). 
Table 1

Beamline optics specifications, measured radii and slope errors.

\begin{tabular}{|c|c|c|c|c|c|c|}
\hline & & Collimating mirror $\dagger \neq$ & Plane mirror $\dagger$ & Plane grating§ & Focusing mirror $\uparrow$ & Refocusing mirror \\
\hline Shape & & Toroidal & Flat & Flat & Cylinder & Toroidal \\
\hline Position & (m) & 16.3 & 21.3 & 21.3 & 22.3 & 29.8 \\
\hline Source distance $h / v$ & (m) & $16.3 / 16.3$ & - & - & $-1 \infty$ & $1.5 / 1.5$ \\
\hline Total deflection angle & $\left({ }^{\circ}\right)$ & 176 & $155-180$ & $155-180$ & 178 & 178 \\
\hline Geometrical surface size & $(\mathrm{mm})$ & $190 \times 40$ & $310 \times 50$ & $100 \times 20$ & $180 \times 40$ & $180 \times 40$ \\
\hline Optical surface size & $(\mathrm{mm})$ & $140 \times 20$ & $275 \times 20$ & $90 \times 15$ & $150 \times 15$ & $100 \times 5$ \\
\hline Footprint $(4 \sigma)$ at $400 \mathrm{eV}$ & $(\mathrm{mm})$ & $100 \times 2$ & & & $100 \times 4$ & $25 \times 1$ \\
\hline Roughness (r.m.s.) & $(\mathrm{nm})$ & 0.1 & 0.15 & 0.16 & 0.161 & 0.374 \\
\hline Coating $(30 \mathrm{~nm})$ & & $\mathrm{Pt}$ & $\mathrm{Pt}$ & $\mathrm{Au}$ & $\mathrm{Pt}$ & $\mathrm{Pt}$ \\
\hline Tangential radius & $(\mathrm{mm})$ & 407000 & - & - & - & 89000 \\
\hline Sagittal radius & $(\mathrm{mm})$ & 1138 & - & - & 209.92 & 26.32 \\
\hline Tangential slope error (r.m.s.) & $(\mu \mathrm{rad})$ & 2.4 & 0.67 & 0.37 & 1.9 & 2.2 \\
\hline Sagittal slope error (r.m.s.) & $(\mu \mathrm{rad})$ & 6.78 & 0.66 & 0.9 & 2.61 & 6.8 \\
\hline Line density & $(1 / \mathrm{mm})$ & - & - & 1200 & - & - \\
\hline
\end{tabular}

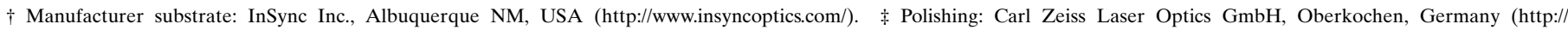
www.zeiss.de/lo). \$ Manufacturer: Carl Zeiss Optronics GmbH, Oberkochen, Germany. @ Manufacturer: WinlightX, Pertuis, France (http://www.winlightx.com/).

The beamline's optical layout is schematically shown in Fig. 3. The optical and geometrical specification for each optical element are given in Table 1 . The beamline front-end is equipped with moving blades, which can be used to change the accepted radiation cone coming from the undulator. The collimating mirror has a toroidal shape used to collimate the light vertically and focus horizontally at the exit slit position (see Fig. 3). This same mirror is used to switch between $\mathrm{X}$-Treme and Phoenix as pointed out in Fig. 1. The vertical focus at the exit slit is made by a cylindrical mirror (focusing

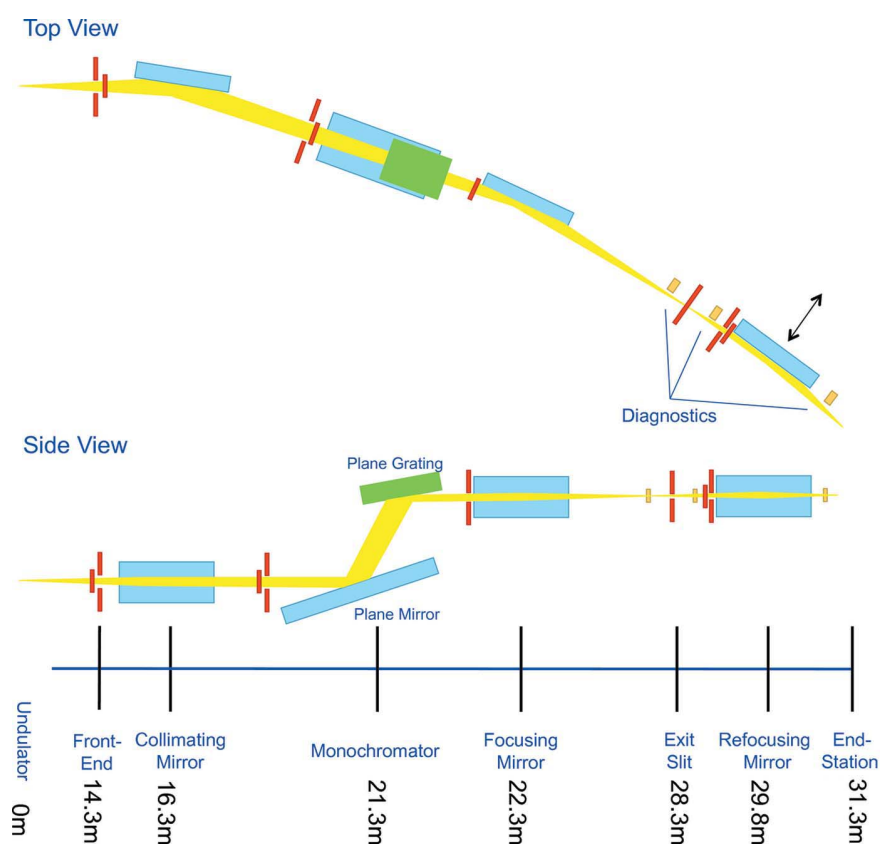

Figure 3

Optical layout of the X-Treme beamline. The optical elements are given in the bottom legend. The red rectangles are adjustable apertures and the orange marks are diagnostics as described in the text. mirror in Fig. 3) located downstream from the monochromator. The dispersion length (distance between exit slit and focusing mirror) is $6 \mathrm{~m}$. The toroidal mirror after the exit slit refocuses the beam both vertically and horizontally at the end-station (refocusing mirror in Fig. 3). The focused spot at the end-station is a 1:1 image of the exit slit. The calculated focused spot size at the sample is $220 \mu \mathrm{m}$ FWHM horizontally while vertically it is the image of the adjustable exit slit opening. The beamline also offers the possibility of a defocused beam at the sample. This feature is mostly used for samples sensitive to radiation damage, since by not focusing the beam the flux density at the sample is reduced. The change between focused and defocused beam is made by retracting the refocusing mirror, as pointed out in Fig. 3. For defocused beam the spot size is $490 \mu \mathrm{m}$ FWHM horizontally and vertically it varies depending on $c_{\mathrm{ff}}$. For $c_{\mathrm{ff}}=2.25$ the defocused vertical spot is $635 \mu \mathrm{m} \mathrm{FWHM}$ and $1.3 \mathrm{~mm} \mathrm{FWHM}$ for $c_{\mathrm{ff}}=5$. Fig. 4 shows the measured focused spot size at the sample position for an exit slit opening of $40 \mu \mathrm{m}$. This image corresponds to $230 \mu \mathrm{m}$ FWHM horizontally and $35 \mu \mathrm{m}$ FWHM vertically.

We point out that another option for the optical layout would have been to perform the horizontal focus by the

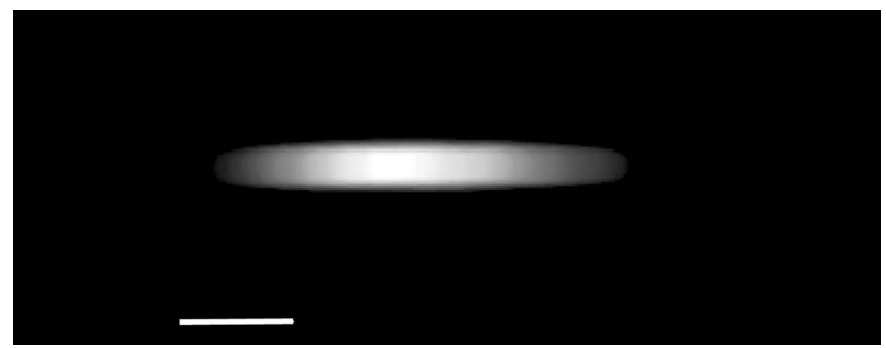

Figure 4

Focused beam spot measured with a CCD camera at the sample position with the vertical exit slit set to $40 \mu \mathrm{m}$. The horizontal bar corresponds to $100 \mu \mathrm{m}$ and the scale is the same for horizontal and vertical directions. 
focusing mirror. This is the design, for example, used in the SIM beamline at SLS (Flechsig et al., 2010) and we will refer to it as FM-focus for simplicity. In our case the collimating mirror is responsible for the horizontal focusing (CM-focus). The primary reason for choosing the CM-focus was that with the FM-focus design it would not have been possible to accept the full beam at the focusing mirror. This difference in acceptance comes from the fact that our monochromator and focusing mirror are further away from the source than in the SIM beamline. This increased distance was necessary to allow enough space for both X-Treme and Phoenix beamlines. In the FM-design the beam is horizontally divergent until the focusing mirror, which together with the increased distance from the source would cause the beam horizontal footprint to be larger than the mirror acceptance. Another difference between both designs is that the FM-focus provides a smaller horizontal spot size than the CM-focus. This increased spot size is not so relevant for $\mathrm{X}$-Treme since it is a beamline dedicated to spectroscopy.

Fig. 3 points out the position of three diagnostics mounted at the beamline: before and after the exit slit and after the refocusing mirror. These diagnostics consist of horizontal linear translation stages where two copper blades with sharp ends in the vertical direction are mounted facing each other forming a fixed vertical aperture. The copper blades are connected to an electrical feedthrough allowing the measurement of the total-electron-yield current between the copper blade and ground, when the blade is positioned in front of the beam. The translation stage is supported by a frame to give stability and is driven by a five-phase motor allowing a minimum step size of $300 \mathrm{~nm}$. The distance between the copper blades and alignment marks positioned outside the linear stage is carefully measured before mounting. This allows the calibration of the blade position according to the theoretical beam path. Therefore, by measuring the electron yield current to the copper blades while scanning their horizontal position, one can measure the position and width of the beam. If a beam imaging chamber is mounted in the endstation position, the copper blades can also be used to analyze the horizontal focus position of the mirrors. Using the blades to cut the beam partially, the image at the end-station will show a cut from the same or opposite side, depending on whether the blade is before or after the focus. Performing this imaging procedure for different angles of the mirrors one can analyze how the focus position changes.

The translation stage length allows mounting more diagnostics besides the copper blades. The two stages after the exit slit are equipped with a photodiode to measure the flux and a gold mesh to measure the beam intensity arriving on the sample, at the same time as the sample measurement. The stage after the refocusing mirror has in addition a test sample containing $\mathrm{La}, \mathrm{Fe}, \mathrm{Co}$ and $\mathrm{O}$, which allows simple characterization of the beamline without the end-station. The diagnostics before the exit slit has a $3 \mu \mathrm{m}$-thick Al filter used to reduce the flux in case there is a problem with radiation damage of the sample.
A further diagnostic tool for the horizontal beam position and width is the analysis of the dispersion line image at the exit slit position. The dispersion line image is formed by a camera looking through a viewport pointing to a fluorescent screen mounted directly above the exit slit.

The beamline is also equipped with three sets of adjustable apertures to reduce the stray light, as shown in Fig. 3. The first double-aperture set is positioned before the monochromator. The second one consists of a vertical aperture and is positioned directly upstream from the focusing mirror. The third one is positioned upstream from the refocusing mirror and consists of a double-aperture set. They are all motorized and controlled remotely. In addition, the blades are coated with phosphor to allow a qualitative diagnostic of the beam image by cameras mounted at view ports directed to the blades. After the focusing mirror there is an additional screen coated with phosphor to allow visualization of the dispersion line to be as close as possible to the monochromator.

Fig. 5 shows the beamline efficiency calculated with the REFLEC program (Schaefers et al., 2002). The blue curve shows the reflectivity for the three mirrors in the beamline, not including the monochromator. The red curve shows the reflectivity coming from the monochromator alone (plane grating and plane mirror) for $c_{\mathrm{ff}}=2.25$ and a laminar grating with $1 / d=1200$ lines $\mathrm{mm}^{-1}$. The monochromator design allows for three gratings to be mounted in parallel. Currently, a 1200 lines $\mathrm{mm}^{-1}$ grating with laminar profile is installed. Gratings with lower line density are foreseen to be installed in the future. These gratings will be optimized for flux rather than resolving power.

The working range of the 1200 lines $\mathrm{mm}^{-1}$ grating in terms of $c_{\mathrm{ff}}$ and photon energy is determined by a combination of different mechanical constraints of the monochromator. The limits imposed by each of these constraints are shown in Figs. 6(a)-6(d) for a wide energy range. In this figure the hatched areas represent the allowed working region. The first two constraints are imposed by the angular range covered by the grating ( $\beta$, Fig. $6 a)$ and mirror $(\theta$, Fig. $6 b)$. The minimum angle reached by the grating $\left(-87.45^{\circ}\right)$ limits the highest

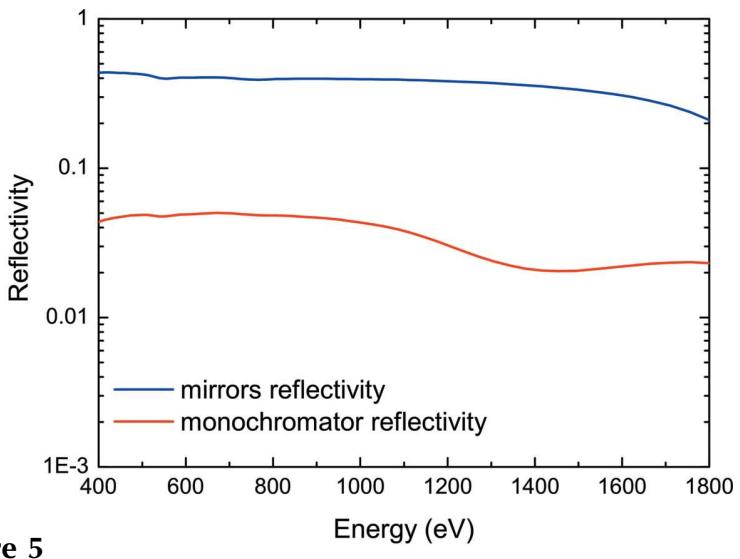

Figure 5

Reflectivity of the three mirrors in the beamline alone (blue curve) and the plane-grating monochromator alone (red curve) for $c_{\mathrm{ff}}=2.25$ and laminar grating with 1200 lines $\mathrm{mm}^{-1}$. 


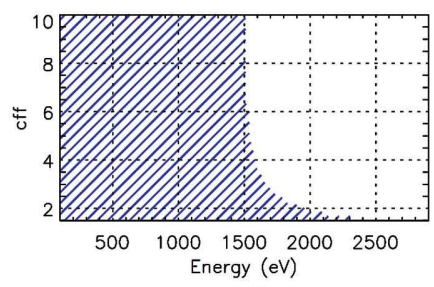

(a)

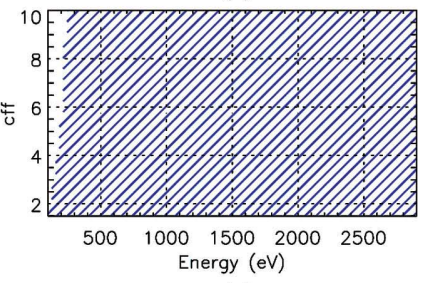

(c)

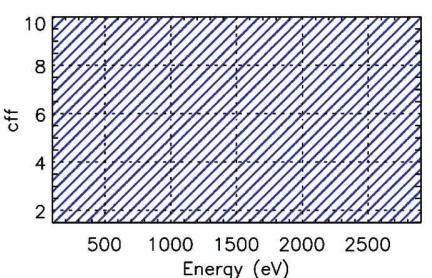

(b)

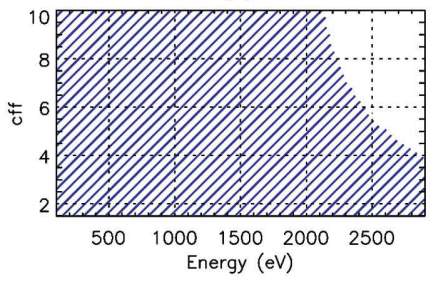

(d)

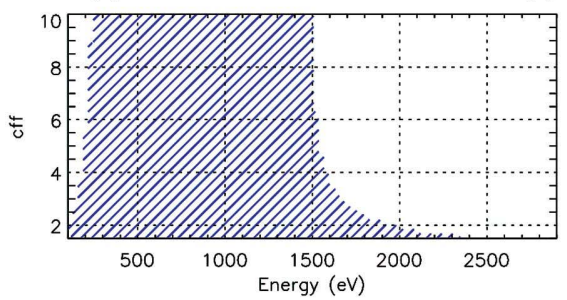

(e)

Figure 6

The hatched areas show the working ranges for the PGM with a 1200 lines $\mathrm{mm}^{-1}$ grating owing to the following mechanical constraints: (a) $-87.45^{\circ}<\beta<-77^{\circ}$. (b) $80^{\circ}<\theta<90^{\circ}$. (c) $\varphi<3^{\circ}$. (d) Plane mirror acceptance of full beam. (e) Combination of all constraints.

energy to $1800 \mathrm{eV}$ for $c_{\mathrm{ff}}=2.25$, as can be seen in Fig. $6(a)$. The mirror angular travel does not impose any limit within the range plotted in Fig. $6(b)$.

The next factor determining the monochromator working range is the full beam acceptance by the plane mirror. The maximum $\theta$ for which the full beam is accepted depends on the vertical distance between the mirror reflecting surface and its center of rotation, schematically shown in Fig. 2. This distance is, in turn, directly proportional to the vertical offset between the plane mirror and the plane grating. The smaller the offset, the higher is the maximum energy cut-off. In the X-Treme monochromator the vertical offset between the plane grating and the plane mirror is $10 \mathrm{~mm}$. As seen in Fig. 6(d), the full beam acceptance is not fulfilled only for energy values above $2000 \mathrm{eV}$ for $c_{\mathrm{ff}}=10$, for example.

The last limit comes from the maximum angle between mirror and grating, $\varphi$, which is also represented in Fig. 2. This angle also depends on the vertical offset between mirror and grating: the smaller the offset, the smaller the maximum allowed $\varphi$ will be. In our case the maximum $\varphi$ is $3^{\circ}$, which only excludes the energy region below $100 \mathrm{eV}$ for $c_{\mathrm{ff}}$ above 4 , as shown in Fig. 6(c).

The combination of all mechanical constraints results in the final working range represented by the hatched area in Fig. 6(e). As seen in this figure, the monochromator mechanics allows operation down to low energy; however, the beamline is optimized for operation starting from $400 \mathrm{eV}$.

The 1200 lines $\mathrm{mm}^{-1}$ grating is optimized for high energy resolution. The calculated resolving power, defined as $\lambda / \Delta \lambda$, is

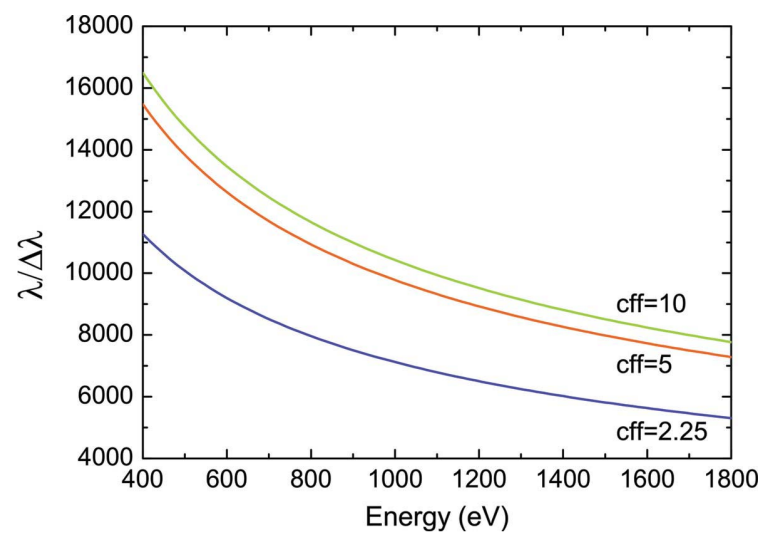

Figure 7

Calculated resolving power as a function of energy for different values of $c_{\mathrm{ff}}$.

plotted in Fig. 7. From this figure one sees that the expected resolving power can be as high as 11000 for $c_{\mathrm{ff}}=2.25$ or 16000 for $c_{\mathrm{ff}}=10$, at $400 \mathrm{eV}$. This calculation is done considering two contributions: (i) the energy resolution coming from the vertical opening of the exit slit, and (ii) the finite vertical size of the monochromatic beam spot. Taking into account our grating illumination, diffraction-limited effects can be neglected. The resulting wavelength bandwidth is calculated as

$$
\Delta \lambda_{\text {total }}=\left(\Delta \lambda_{\text {slit }}^{2}+\Delta \lambda_{\text {spot }}^{2}\right)^{1 / 2}
$$

Each contribution in (5) is calculated using the following expression,

$$
\Delta \lambda=\cos \beta(s / N D),
$$

where $D$ is the dispersion distance $(6000 \mathrm{~mm})$ and $N$ is the grating density $\left(1200\right.$ lines $\left.\mathrm{mm}^{-1}\right)$. In the case of $\Delta \lambda_{\text {slit }}, s$ is the exit slit vertical opening while, for $\Delta \lambda_{\text {spot }}, s$ is considered as the FWHM of the monochromatic beam spot size. The beam spot size is calculated using the ray-tracing program Phase (Bahrdt et al., 1995), considering the measured slope errors as given in Table 1. The values found are (FWHM): $12 \mu \mathrm{m}, 13 \mu \mathrm{m}$ and $18 \mu \mathrm{m}$ for $c_{\mathrm{ff}}=10,5$ and 2.25 , respectively. The exit slit size used in the calculation is $10 \mu \mathrm{m}$. Since the exit slit opening used is smaller than the spot size, Fig. 7 mostly reflects the spot size limited resolving power.

\section{Commissioning measurements}

\subsection{Energy resolution}

In order to characterize the energy resolving power, absorption spectra of different gases were measured using a gas cell permanently installed in the beamline directly after the exit slit. The nitrogen $K$-edge spectrum measured for $\mathrm{N}_{2}$ gas is shown in Fig. 8. This spectrum corresponds to the transition $\mathrm{N} 1 s \rightarrow \pi^{*}$, which occurs around $400 \mathrm{eV}$. The measurement was made with $c_{\mathrm{ff}}=10$ and an exit slit opening of $10 \mu \mathrm{m}$. The shape of the absorption peaks can be described by Voigt functions, which consist of the convolution of a Lorentzian function describing the finite life time of the $\mathrm{N} 1 s$ 


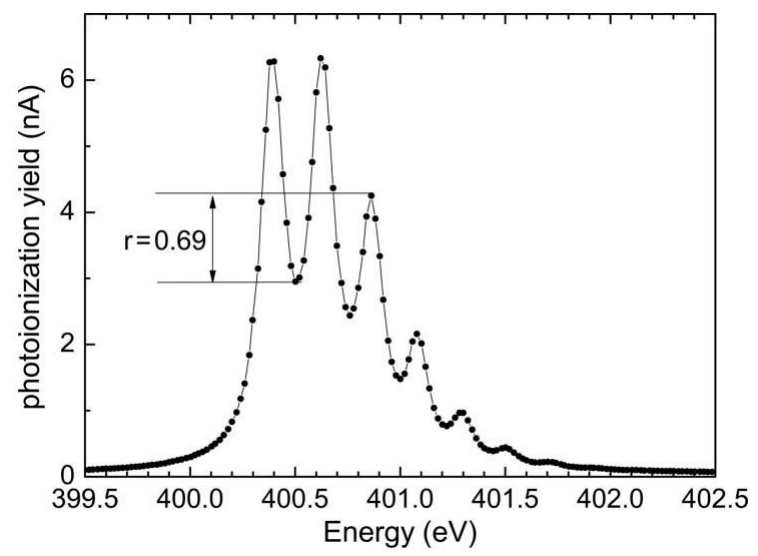

Figure 8

Measured $\mathrm{N}_{2}$ gas spectrum at the $\mathrm{N} K$-edge, with an exit slit opening of $10 \mu \mathrm{m}$ and $c_{\mathrm{ff}}=10$.

core hole, and a Gaussian function describing the energy bandwidth. The spectrum in Fig. 8 was fitted to seven Voigt functions, keeping the life time broadening fixed to $112 \mathrm{meV}$, which is the average among the varying values in the literature (Kato et al., 2007a). The resulting Gaussian FWHM obtained from the fit is $50 \mathrm{meV}$. This gives a resolving power of $400 \mathrm{eV} /$ $50 \mathrm{meV}=8000$. This value is below the one expected from the calculation shown in Fig. 7. However, we attribute this largely to the uncertainty in the value of the $\mathrm{N} 1 s$ natural line width. An alternative method avoiding this problem is based on the ratio between the second valley and the third peak in the $\mathrm{N}_{2}$ spectra, as introduced by Chen \& Sette (1989). High energy resolving power implies low overlap of the spectroscopic features and therefore low ratios. The reported values for different beamlines vary from 0.9 to 0.66 (Chen \& Sette, 1989; Petersen et al., 1995; Follath et al., 2004; Flechsig et al., 2010). We find a ratio of 0.69 comparable with the best value of 0.66 which has been associated with a resolving power above 10000 (Follath et al., 2004).

The energy resolution was further characterized around $800 \mathrm{eV}$ by measuring the Ne $K$-edge for neon gas, as shown in Fig. 9. This measurement was made with $c_{\mathrm{ff}}=5$ and an exit slit opening of $10 \mu \mathrm{m}$. Fitting the main peak (at $864.7 \mathrm{eV}$ ) to a

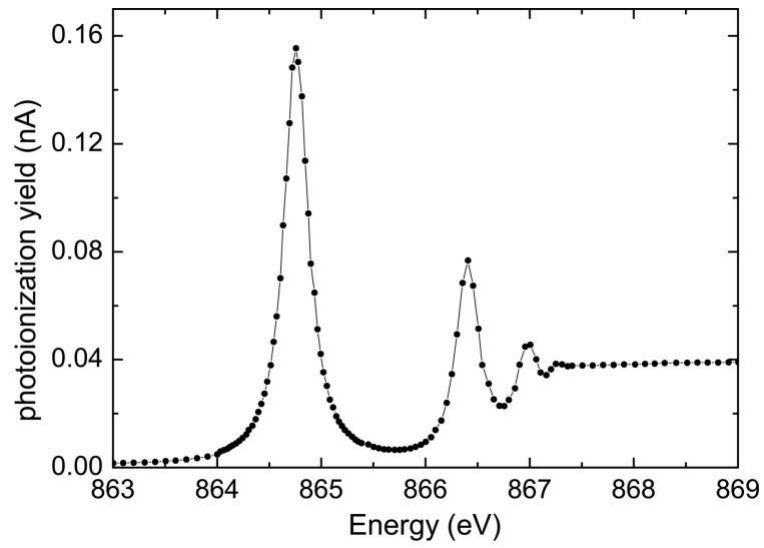

Figure 9

Measured $\mathrm{Ne}$ gas spectrum at the $\mathrm{Ne} K$-edge, with an exit slit opening of $10 \mu \mathrm{m}$ and $c_{\mathrm{ff}}=5$.
Voigt function gives a total FWHM of $280 \mathrm{meV}$. Considering the $\mathrm{Ne} 1 \mathrm{~s}$ core hole contribution to be $252 \mathrm{meV}$ (Kato et al., 2007b), the resulting Gaussian contribution is $90 \mathrm{meV}$ (FWHM). This corresponds to a resolving power of $865 \mathrm{eV} /$ $90 \mathrm{meV}=9600$, which is close to the calculated value of 10900 .

\subsection{Polarization}

The Apple II undulator can deliver light with different polarization settings: circular left or right and linear light varying from $0^{\circ}$ to $90^{\circ}$. The degree of circular polarization depends on the ratio $K_{x} / K_{y}$, where the parameter $K$ is defined as (Clarke, 2004)

$$
\begin{aligned}
K & =0.934 B \lambda_{\mathrm{U}}, \\
K^{2} & =K_{x}{ }^{2}+K_{y}{ }^{2} .
\end{aligned}
$$

In (7), $B$ is the magnetic field created by the undulator in Teslas and $\lambda_{\mathrm{U}}$ is the undulator period in centimeters. In the first harmonic, $K_{x} / K_{y}$ is always equal to 1 leading to $100 \%$ circular polarization. The degree of polarization in the first harmonic for the X-Treme undulator was verified by comparing an XMCD measurement of a Co thin film capped with Pt measured at the X-Treme and SIM beamlines under the same experimental conditions. The XMCD intensity measured at the two beamlines normalized by the XAS is the same within $5 \%$. The degree of polarization at the SIM beamline was measured with a polarimeter from BESSY (Schäfers et al., 1999) and found to be higher than $97 \%$ (Flechsig et al., 2010).

For the X-Treme undulator, the maximum energy achieved with the first harmonic is $1000 \mathrm{eV}$. Above this energy one needs to use the third harmonic, where $100 \%$ circular polarization cannot be achieved and the light is linearly or elliptically polarized. The degree of circular polarization when operating in elliptical mode depends on the ratio $K_{x} / K_{y}$ defined by (7) and (8). We have measured the degree of circular polarization by measuring the XMCD of the same test sample for varying $K_{x} / K_{y}$ ratios in the third harmonic and comparing with $K_{x} / K_{y}=1$ measured in the first harmonic. Since the measurements were made on the same sample under the same applied magnetic field, the intensity of the XMCD signal, normalized by the XAS, is linearly proportional to the degree of circular polarization. In Fig. 10 the black squares show the XMCD intensity normalized by the value in the first harmonic. The full curve shows the calculated degree of circular polarization defined as $S_{3} / S_{0}$, where $S_{3}$ and $S_{0}$ are the Stokes parameters (Clarke, 2004). The measured and calculated values show a good agreement, if we consider the light polarization to be $100 \%$ in the first harmonic. The larger the degree of circular polarization in the third harmonic, the smaller is the photon flux, as can be seen by the blue circles in Fig. 10 showing the beam intensity normalized by the value in the first harmonic.

The data plotted in Fig. 10 can be used to create the figure of merit, defined as the degree of circular polarization squared times the flux. The result is shown in Fig. 11. The figure of 


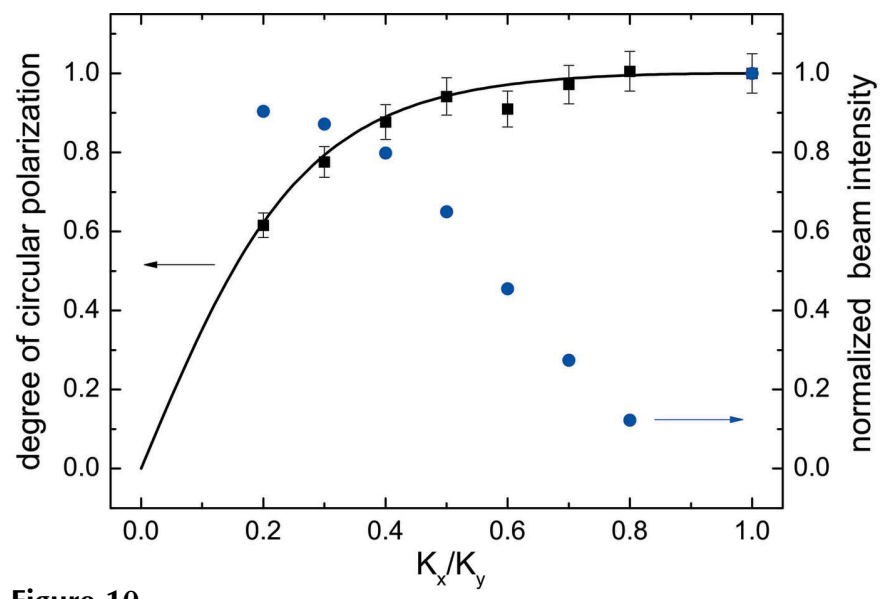

Figure 10

Left axis: calculated (solid line) and measured (black squares) degree of circular polarization. Right axis: blue circles show measured incoming beam intensity. Both measured values have been normalized to the value measured in the first harmonic.

merit is maximum for $K_{x} / K_{y}=0.4$, as found before in a similar Apple II undulator (Bahrdt et al., 2001).

Currently, in the third harmonic the undulator is set for $K_{x} / K_{y}=0.8$, where the degree of circular polarization is $99.5 \%$ and the flux is around ten times smaller than in the first harmonic. This is the setting used for all data presented here. A second mode optimizing the figure of merit, with $K_{x} / K_{y}=$ 0.4 , is also available, where the degree of circular polarization is around $90 \%$ and the photon flux is $80 \%$ of that in the first harmonic.

\subsection{Flux}

The beamline flux was measured using a Si photodiode with an area of $10 \mathrm{~mm} \times 10 \mathrm{~mm}$ placed after the last optical element, thereby monitoring the actual flux reaching the sample. In order to obtain the absolute flux in number of photons per second, the measured photocurrent is multiplied by the photodiode quantum efficiency (Gullikson et al., 1996). Figs. 12-14 show the measured flux for different beamline

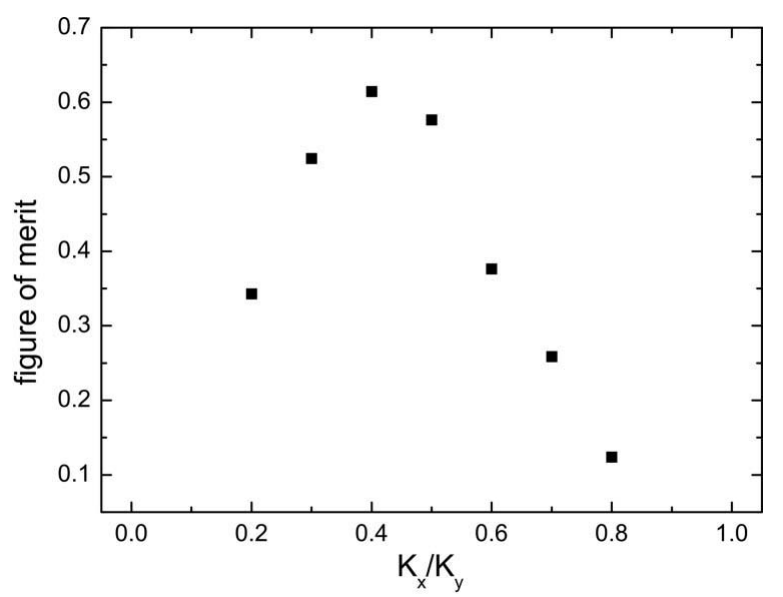

Figure 11

Figure of merit versus $K_{x} / K_{y}$ ratio. The figure of merit is calculated as the degree of circular polarization squared times the incoming total flux.

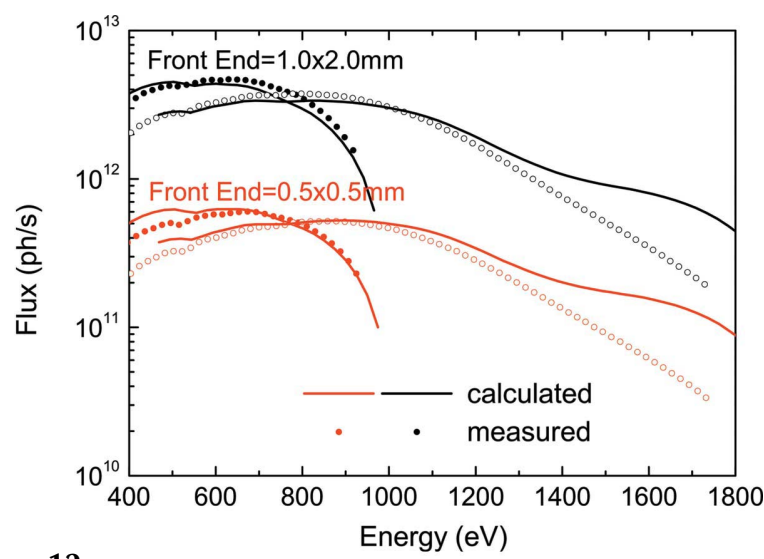

Figure 12

Flux measured after the last optical element for linear horizontal light, for the first (closed symbols) and third (open symbols) harmonics and two different front-end openings: $0.5 \mathrm{~mm} \times 0.5 \mathrm{~mm}$ (red) and $1.0 \mathrm{~mm} \times$ $2.0 \mathrm{~mm}$ (black). The exit slit opening was $50 \mu \mathrm{m}$ and $c_{\mathrm{ff}}=2.25$. The calculated flux is shown by the full lines.

settings. The highest flux of $4.7 \times 10^{12}$ photons $\mathrm{s}^{-1}$ occurs for photon energies between 600 and $700 \mathrm{eV}$.

The comparison between the measured and calculated flux with linear horizontal polarization is shown in Fig. 12. The undulator flux for different front-end openings was calculated using the SRW software (Chubar \& Elleaume, 1998). The calculation result is then multiplied by the beamline efficiency presented in Fig. 5 and corrected by the actual energy bandwidth for the measurement condition. The agreement between expected and measured flux is excellent, except for energy values above $1400 \mathrm{eV}$, where the measured flux is lower than the calculated one by up to a factor of two. The same behavior is observed for different front-end openings.

The flux variation with light polarization in the first and third harmonics is illustrated by the measurements presented in Fig. 13. This figure shows the flux measured for linear horizontal, linear vertical, circular plus and circular minus polarized light. Another factor influencing the flux reaching the sample is the $c_{\mathrm{ff}}$ parameter of the monochromator. In

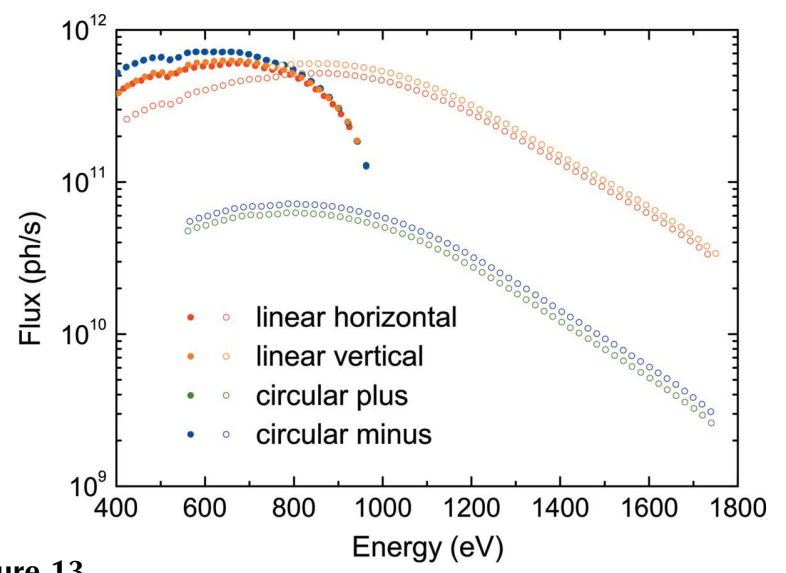

Figure 13

Flux measured after the last optical element for four different polarization settings in the first (closed symbols) and third (open symbols) harmonics. The front-end and exit slit openings were $0.5 \mathrm{~mm} \times$ $0.5 \mathrm{~mm}$ and $50 \mu \mathrm{m}$, respectively, and $c_{\mathrm{ff}}=2.25$. 


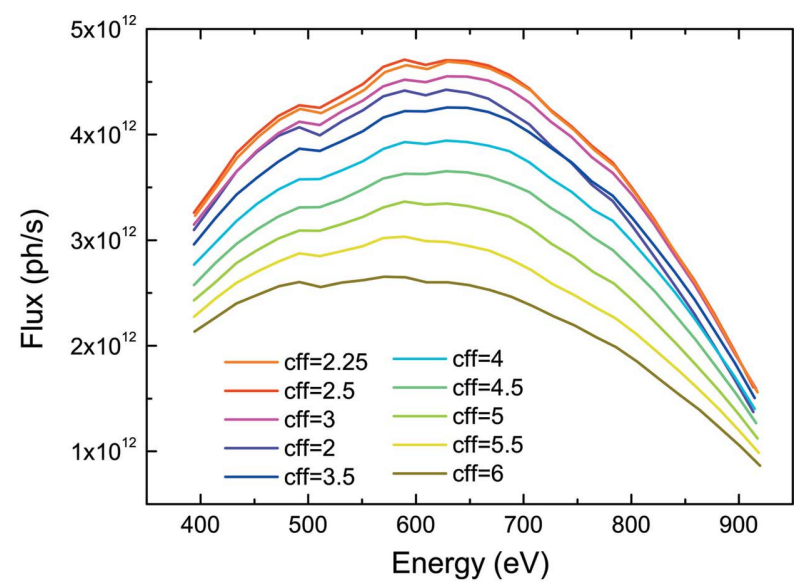

Figure 14

Flux measured after the last optical element for different values of $c_{\mathrm{ff}}$ in the first harmonic of the undulator. The front-end and exit slit openings were $1.0 \mathrm{~mm} \times 2.0 \mathrm{~mm}$ and $50 \mu \mathrm{m}$, respectively.

order to illustrate this effect, flux measurements using the first harmonic for varying values of $c_{\mathrm{ff}}$ are shown in Fig. 14. From this figure it is seen that the highest flux is achieved for $c_{\mathrm{ff}}$ values between 2.25 and 2.5. The measurements shown in Figs. 12 and 13 were made with a $c_{\mathrm{ff}}$ value of 2.25 .

As mentioned earlier, the possibility to freely vary $c_{\mathrm{ff}}$ gives the flexibility to set the monochromator for highest flux or resolving power. The exit slit opening has a similar effect with the difference that the focused beam spot size changes as well. The trade-off between energy resolution and flux for different settings of $c_{\mathrm{ff}}$ and exit slit openings is illustrated in Fig. 15. In this figure the resolving power was extracted from the Voigt fit of measured $\mathrm{N}_{2}$ spectra, as explained in \$3.1. The flux in photons $\mathrm{s}^{-1}$ for the same settings is also plotted. These measurements show that the best energy resolution is achieved for high $c_{\mathrm{ff}}$ values of 5 or 10 . On the other hand, the flux for $c_{\mathrm{ff}}=10$ is $30 \%$ lower than for $c_{\mathrm{ff}}=5$. The highest flux is obtained for $c_{\mathrm{ff}}=2.25$, as already expected from the measurement shown in Fig. 14. The PGM setting with $c_{\mathrm{ff}}=1.5$ has low resolving power and flux. However, this is the setting

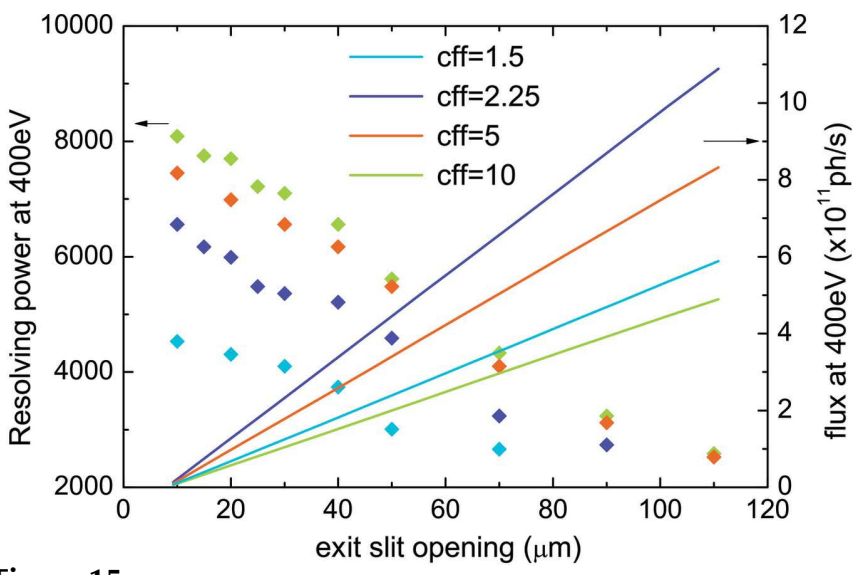

\section{Figure 15}

Measured resolving power and photon flux as a function of exit slit opening and for different values of $c_{\mathrm{ff}}$. Left axis: energy-resolving power (symbols) estimated from $\mathrm{N}_{2}$ spectra measurements. Right axis: flux measured at $400 \mathrm{eV}$ (continuous line). for best higher harmonic rejection as discussed in the next section.

\subsection{Higher-order rejection}

Equation (4) shows that for the same angle of the monochromator and grating different diffraction orders are allowed, represented by the integer $m$. In most experiments one is interested only in the first diffraction order $(m=1)$; however, the higher diffraction orders are still allowed. These higher orders will come as two or three times the set energy, for second- and third-order diffraction, respectively. In this section we discuss how large the contributions of these higher orders are and how they can be suppressed.

The higher-order contamination (HIOC) is defined as the flux coming from the second- and third-order diffraction divided by the total flux, or, in other words, as

$$
\text { HIOC }=\frac{\text { Flux }(2 \text { nd order }+3 \text { rd order })}{\text { Flux }(1 \text { st order }+2 \text { nd order }+3 \text { rd order })} .
$$

Fig. 16(a) shows the calculated HIOC plotted versus energy for $c_{\mathrm{ff}}=2.5$ and 1.5. For $c_{\mathrm{ff}}=2.5$ the HIOC is around $3 \%$ for photon energy below $600 \mathrm{eV}$ and rapidly decreases above this energy. In Fig. 16(b) an X-ray absorption measurement taken on a Co- and La-containing sample illustrates what a HIOC of $3 \%$ means in practice. The blue curve refers to the bottom/left energy/intensity axes and shows an absorption measurement in the energy range from 760 to $860 \mathrm{eV}$. In this curve one identifies the Co $L_{3,2}(775 \mathrm{eV}$ and $791 \mathrm{eV})$ and $\mathrm{La} M_{5,4}(831 \mathrm{eV}$ and $847 \mathrm{eV}$ ) absorption edges. The red curve, to which the top/ right energy/intensity axes refer, shows for the same sample the measurement in the energy range between 380 and $430 \mathrm{eV}$. In this energy range there are no absorption edges for any of the elements composing the sample; however, one can clearly observe the Co and La edges at half their energies. This is evidence for the higher-order contamination at these low energies when using $c_{\mathrm{ff}}=2.25$.

The possibility to change the $c_{\mathrm{ff}}$ value, however, allows the higher-order contamination to be almost eliminated. The curve with red circles in Fig. 16(a) shows that the calculated HIOC for $c_{\mathrm{ff}}=1.5$ is below $0.1 \%$. In Fig. $16(b)$ an X-ray absorption spectrum for the same sample measured with $c_{\mathrm{ff}}=$ 1.5 is plotted with red symbols. The measurement was made in the low energy range from 380 to $430 \mathrm{eV}$ and, as one can see, it is entirely free of higher harmonic contributions. Therefore, for cases where HIOC can be a problem, a $c_{\mathrm{ff}}$ value of 1.5 is the most appropriate setting for the monochromator.

\subsection{End-station}

The end-station specifications are summarized in Table 2. The chamber with the magnets, as well as the variabletemperature sample insert, were purchased from Scientific Magnetics. $^{3}$ The cryostat houses two split-coil pairs of NbTi

\footnotetext{
${ }^{\mathbf{3}}$ The variable-temperature sample insert and the magnets were constructed by Scientific Magnetics, Abingdon, UK (http://www.scientificmagnetics.com/). The vacuum chamber was manufactured by CVT Ltd, Milton Keynes, UK (http://www.cvt.ltd.uk/).
} 
Table 2

End-station specifications.

Maximum field along the beam $(x)$

Maximum field perpendicular to the beam $(z)$

Maximum field for each coil during vectorial operation

Maximum sweep velocity $x$

Maximum sweep velocity $z$

Maximum sweep velocity vector field mode

Sample temperature

Liquid-helium bath total capacity

Liquid-helium bath refill volume

Liquid-nitrogen bath capacity

Hold time of liquid nitrogen and liquid helium

superconducting wire. One pair of coils generates a field up to $7 \mathrm{~T}$ along the beam direction $(x)$; the other pair generates a field of up to $2 \mathrm{~T}$ perpendicular to the beam in the horizontal plane $(z)$. Both coils can be operated simultaneously, allowing a vector field in the horizontal plane $x z$. During vector field operation the maximum field generated by each coil is $2 \mathrm{~T}$.
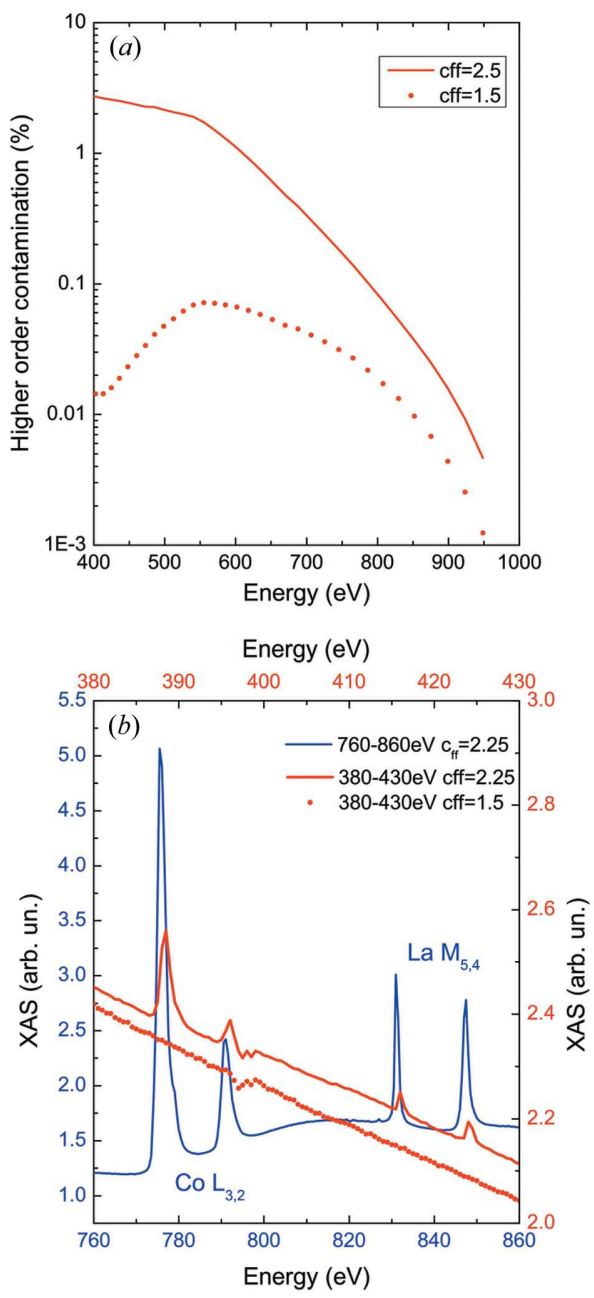

Figure 16

Higher-order contamination for different $c_{\mathrm{ff}}$ values. (a) Calculation for $c_{\mathrm{ff}}=2.5$ (line) and 1.5 (symbols). Note the logarithmic scale for the HIOC.

(b) X-ray absorption measurement of a sample containing Co and La. Blue line: measurement between 760 and $860 \mathrm{eV}$, plotted against the left/ bottom intensity and energy axes. Red curves: measurements for $c_{\mathrm{ff}}=2.25$ (line) and 1.5 (symbols), in the energy range $380-430 \mathrm{eV}$, plotted against the right/top intensity and energy axes.
The $7 \mathrm{~T}$ coils, when operated alone, have a maximum sweep velocity of $2 \mathrm{~T} \mathrm{~min}^{-1}$. The $z$-coil alone, as well as the vector field mode, has a sweep velocity of $0.36 \mathrm{~T} \mathrm{~min}^{-1}$. Magnetization curves with steps of $2 \mathrm{mT}$ have been measured. A minimum resolution of $10^{-4} \mathrm{~T}$ during continuous ramping has been achieved. The remanent field measured at the sample varies between 4 and $10 \mathrm{mT}$ depending on the maximum field used. The field homogeneity is $1 \%$ over $10 \mathrm{~mm}$ in any direction from the center of the bore.

The sample insert is a pumped ${ }^{4} \mathrm{He}$ cryostat. Liquid helium is transferred from the magnet main bath to the sample insert $4 \mathrm{~K}$ pot through a siphon external to the cryostat. This is done by under-pressurizing the sample insert $4 \mathrm{~K}$ pot with respect to the magnet bath. A motorized needle valve separates the $4 \mathrm{~K}$ pot from a $1 \mathrm{~K}$ pot. The small reservoir of the $1 \mathrm{~K}$ pot reaches $1.4 \mathrm{~K}$ when pumped. The lowest temperature measured directly at the sample position is $2.0 \mathrm{~K}$. Stabilization at higher temperatures is achieved by controlling a heater and the needle valve opening.

In order to achieve the lowest temperature, the sample shielding from infrared radiation is an important issue. The vacuum chamber surrounding the sample is at liquid-nitrogen temperature and there are six access ports. The top port is used for mounting the variable-temperature sample insert and the bottom port is used for sample transfer. On the bottom port there is a double layer sliding infrared shield, at liquidhelium and liquid-nitrogen temperature, which is closed during measurement. In one of the four ports at the beam height a sapphire window acts as an infrared shield and allows optical visualization of the sample. The two ports at the beam propagation direction are equipped with sliding shields at liquid-nitrogen temperature. These shields have small openings to accept the incoming X-rays and allow the detection of transmitted X-rays. In case necessary, the infrared shield around the sample can be completely closed by mounting thin metal foils allowing the transmission of X-rays through these openings. The fourth port at beam height is also equipped with a sliding shutter at liquid-nitrogen temperature. This shutter is normally closed but the system can be operated with the shutter open, e.g. in order to direct an additional detector to the sample, of course under the sacrifice of an increased sample temperature. The pressure in the sample space, when the magnet is cooled with liquid helium, is in the low $10^{-11}$ mbar range.

The sample has an electrical contact connected to a SMA feedthrough allowing X-ray absorption detection through the total-electron-yield current measured between sample and ground. The sample insert has four additional electrical contacts connecting the sample holder to a multi-pin electrical feedthrough. These contacts can be used for temperature sensor and Hall probe reading at the sample position. It also allows additional in situ options such as the application of any voltage below $300 \mathrm{~V}$.

Currently, the absorption signal can be detected by total electron yield, transmission or fluorescence. The transmission signal is detected by a photodetector mounted in the back port. The fluorescence signal is detected by a photodiode 
mounted in a translation stage at $90^{\circ}$ with the incoming beam, opposite to the sapphire window mentioned above. For the transmission signal detection it is enough to have the photodiode mounted behind the sliding shutters, and the infrared shield is partially closed, except for the $5 \mathrm{~mm}$ opening left for the transmitted beam. For fluorescence measurements, since the fluorescence yield is small, the photodiode is mounted in a translation stage so that it can be brought close to the sample. As a consequence, the sliding shutter at that port has to be completely open. This will lead to a base temperature higher than $2 \mathrm{~K}$. Both diodes can be mounted/dismounted without venting the main chamber, since there are manual valves on both ports to separate the vacuum between the chamber and the photodiode mounting. There are plans to install a luminescence detector to allow transmission measurements of thin films by detecting the optical luminescence of the substrate (Kallmayer et al., 2007). The intensity of the beam arriving at the sample can be monitored in three places: by a gold mesh before or after the refocusing mirror or by the total-electronyield current of the mirror itself.

A diagram of the end-station is shown in Fig. 17. There are four motorized movements possible for the sample:

(i) rotation of the sample insert around the vertical axis by $355^{\circ}$;

(ii) vertical translation of the sample insert by $50 \mathrm{~mm}$;

(iii) horizontal movement of the whole end-station by $80 \mathrm{~mm}$;

(iv) rotation of the whole end-station around the sample position by $10^{\circ}$.

For the first two movements the cryostat containing the magnets is fixed and only the sample insert moves with respect to the magnets and the incoming beam. The last two movements are possible through a motorization of the end-station frame with respect to the platform fixed on the floor. Therefore, in the case of movements (iii) and (iv), the whole cryostat plus sample insert moves with respect to the incoming beam. Notice that, owing to the restricted space inside the magnet

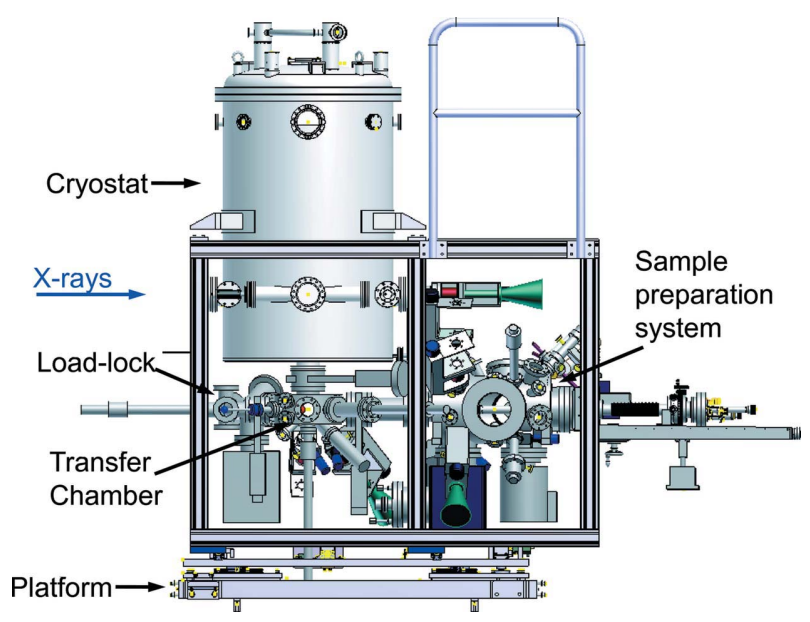

Figure 17

Drawing of the end-station side view showing the sample preparation system, load-lock, transfer chamber and the main cryostat where the measurements are performed.

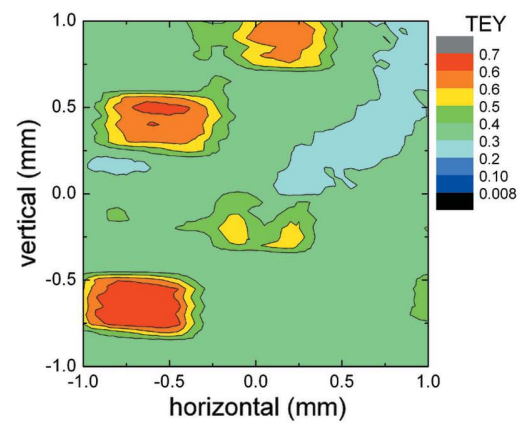

(a)

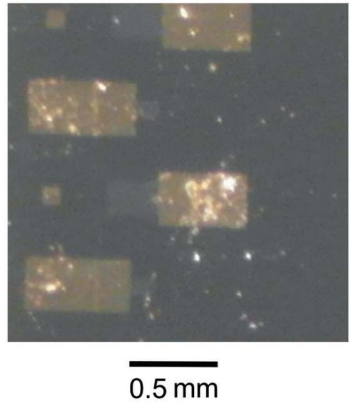

(b)

\section{Figure 18}

(a) Two-dimensional map of a sample prepared by lithography, using the vertical and horizontal motorization of the end-station. The color code comes from the total electron yield (TEY) measured from the sample. (b) Optical image of the same region of the sample. The rectangular gold contacts in $(b)$ are represented in red/orange in $(a)$.

bore $(40 \mathrm{~mm})$, the only way to position the sample horizontally is by moving the whole end-station. Movements (i) to (iii) are routinely used to align the sample with respect to the incoming beam. The motorization of these three movements makes the alignment of small samples easier, as exemplified by Fig. 18. This figure shows a total-electron-yield measurement of a lithographically prepared sample while scanning its horizontal and vertical positions. Through this scan one can easily locate the structures of a few hundred micrometers. An optical image of the sample is also shown in Fig. 18 for comparison.

As mentioned in $\S 2$, the user can opt for a defocused beam spot at the sample, allowing the flux density to be reduced for samples which are sensitive to the X-ray radiation. This is achieved by retracting the refocusing mirror out of the beam. As a consequence of the mirror retraction, the beam path changes by $2^{\circ}$ between focused and defocused beam conditions resulting in a horizontal displacement of $52 \mathrm{~mm}$ at the sample position. In order to re-align the sample to the defocused beam, movements (iii) and (iv) of the end-station frame are used. The change between focused and defocused beam can be made in about $15 \mathrm{~min}$.

One more possible movement of the end-station is the rotation of the whole system out of the beam. This movement allows a second end-station to be connected to the beamline without the need of passing the beam through the cryostat, as shown in Fig. 19. The rotation of the end-station out of the beam is performed using air cushions installed under the endstation platform.

The end-station is also equipped with an in situ sample preparation system, with atomic and molecular evaporators, ion sputter gun, LEED, heating/cooling stage for sample preparation (30-1800 K) and a variable-temperature scanning tunnelling microscope (VT STM XA 50/500) combined with the Qplus AFM package from Omicron. As seen in Fig. 17, the sample preparation system is connected to the vacuum of the cryostat through the transfer chamber which is always kept at ultra-high-vacuum pressure. Ex situ samples are loaded through the load-lock and transferred to the cryostat via the transfer chamber. 
(a)

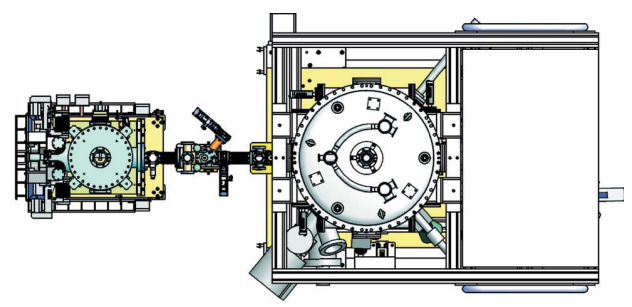

(b)

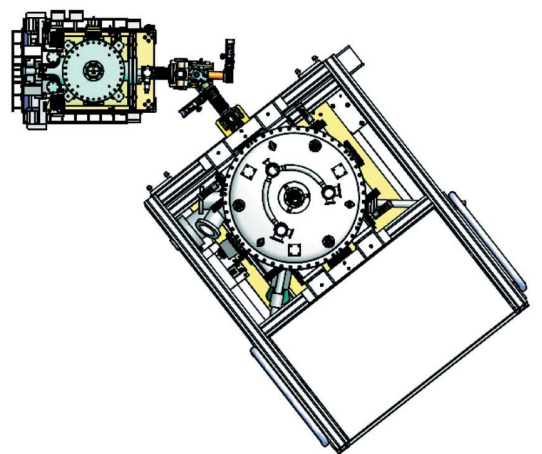

Figure 19

Top view diagram showing $(a)$ the X-Treme end-station on-line and $(b)$ rotated off-line for the connection of another end-station to the second valve via a $2 \mathrm{~m}$-long tube (not shown).

\section{Research examples}

In the following sections we show two research examples carried out at the X-Treme beamline. The first concerns highfield low-temperature XMCD measurements on a Dy-Cr-Dy single-molecule magnet. The second example shows temperature-dependent zero-field XMLD measurements in epitaxial $\mathrm{SmFeO}_{3}$ films through the spin reorientation transition.

All the XAS data presented here were measured using onthe-fly scan mode, where the monochromator and the insertion device are continuously scanned (Krempaský et al., 2010). Using this mode, a spectrum measured over $50 \mathrm{eV}$ takes between 1 and $3 \mathrm{~min}$. When measuring dichroic data, the time to change polarization or magnetic field are crucial. Since the signal consists of the difference between two measurements with opposite magnetic field or polarization, the closer in time these two measurements are taken the smaller is the likelihood of spurious contribution coming from drifts. For the $\mathrm{X}$-Treme undulator, the time to change between left and right circularly polarized light is $30 \mathrm{~s}$.

\subsection{XMCD on a molecular magnet}

Single-molecule magnets are metal-organic molecules containing magnetic ions exchange coupled to each other. These systems present a slow magnetization relaxation, showing a hysteresis if the measurement temperature is below the blocking temperature. In this section we give a brief account of our results on the fluoride-bridged trimer Dy-CrDy molecular magnet (Dreiser et al., 2012).

Fig. 20 shows the XAS measured at the Dy $M_{5,4^{-}}$and $\mathrm{Cr}$ $L_{3,2}$-edges, as well as the respective XMCD signal measured at $6 \mathrm{~T}$. The XAS spectra show good agreement with multiplet
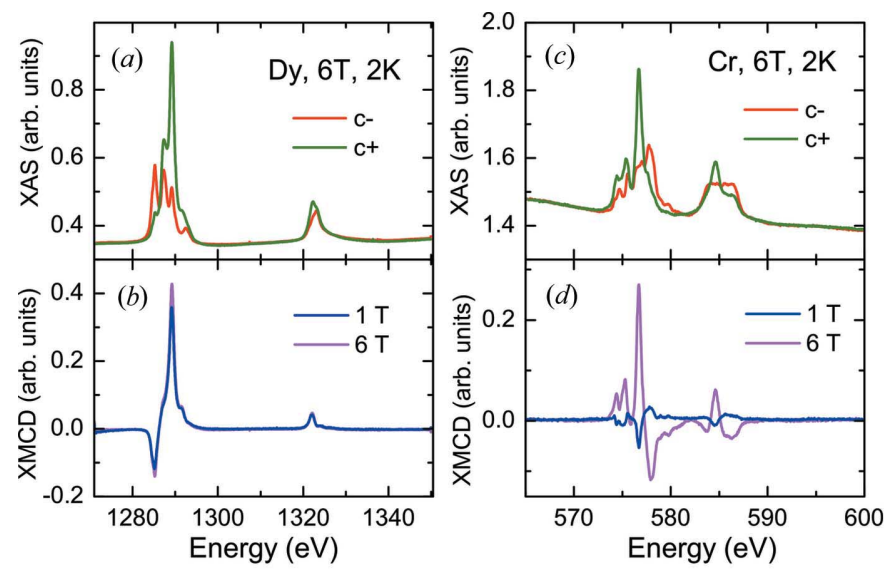

Figure 20

X-ray absorption spectra for circularly polarized light measured at $2 \mathrm{~K}$ and $6 \mathrm{~T}$ at the (a) Dy $M_{5,4^{-}}$and (c) $\mathrm{Cr} L_{3,2^{-}}$edges. The corresponding XMCD data measured at 1 Tand 6 T are shown in panels $(b)$ and $(d)$. This figure is based on Figs. 2 and 3 from Dreiser et al. (2012) and reproduced with permission of The Royal Society of Chemistry.

theory for $\mathrm{Dy}^{3+}$ and $\mathrm{Cr}^{3+}$ (Dreiser et al., 2012). Moreover, from the comparison of the XMCD shape and sign with the simulations we infer that at $6 \mathrm{~T}$ both Dy and $\mathrm{Cr}$ are aligned parallel to the field. Comparing the XMCD measured for Dy between $1 \mathrm{~T}$ and $6 \mathrm{~T}$, one sees that the difference in peak intensity is small and the spectral shape is the same, showing that Dy is almost saturated already at $1 \mathrm{~T}$. On the other hand, for $\mathrm{Cr}$ the XMCD signal changes sign between $1 \mathrm{~T}$ and $6 \mathrm{~T}$, evidencing that the $\mathrm{Cr}$ magnetic moment changes orientation from a ferrimagnetic alignment with respect to Dy at low field to a ferromagnetic one at high field.

In order to study this effect in more detail, element-specific magnetization curves were measured. This was done by measuring the XMCD intensity as a function of magnetic field. Technically this is achieved by measuring the absorption at two energies: at the XMCD peak and before the peak for a background measurement. The electron yield for these two energies is measured for a complete field sweep and repeated for plus and for minus circularly polarized light. The result is presented in Fig. 21 and confirms the interpretation of the XMCD spectra. Cr exhibits a wiggle-shape magnetization showing that it is ferrimagnetically aligned to Dy in its ground state, but at sufficiently high fields the magnetic moment changes direction and points parallel to the applied field. The fit of this magnetization curve with a spin Hamiltonian gives the exchange coupling between Dy and Cr [for more details see Dreiser et al. (2012)]. The black lines in Fig. 21 show the result of such a fit. From the XMCD and XAS spectra shown in Fig. 20 one can obtain the values for spin and orbital moments by applying sum rules (Thole et al., 1992; Carra et al., 1993). This has been done for Dy and the results are plotted as black squares in Fig. 21.

\subsection{Magnetic linear dichroism in $\mathrm{SmFeO}_{3}$}

Orthoferrites present the chemical formula $R \mathrm{FeO}_{3}$, where $R$ is a rare earth, and the structure is an orthorhombically 

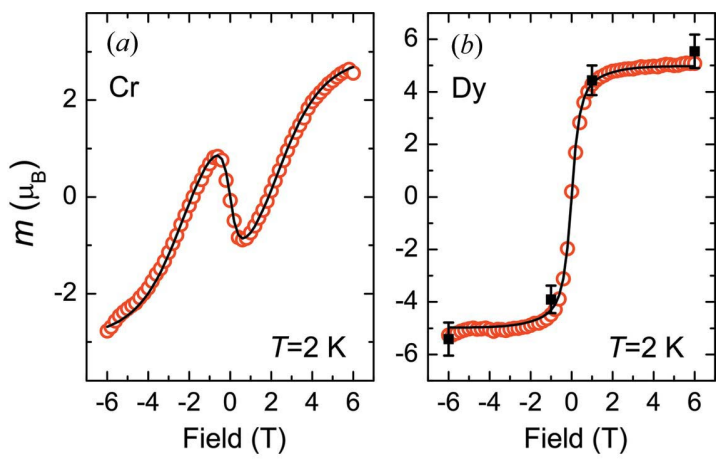

Figure 21

Element-specific magnetization measured for (a) Cr and (b) Dy. Red circles show the measurements while the full lines are fits using a spin Hamiltonian. Black squares are the result from XMCD sum rules. Figures are taken from Dreiser et al. (2012) and reproduced with permission of The Royal Society of Chemistry.

distorted perovskite. The magnetic moments of the $\mathrm{Fe}^{3+}$ ion order antiferromagnetically. Many of these systems present a spin reorientation transition with temperature, where the $\mathrm{Fe}$ spins rotate by $90^{\circ}$ with respect to the crystallographic structure (White, 1969; Bozorth et al., 1956). $\mathrm{SmFeO}_{3}$ is a member of this family with an antiferromagnetic ordering temperature of $T_{\mathrm{N}}=650 \mathrm{~K}$ and a spin reorientation occurring around $480 \mathrm{~K}$ $\left(T_{\mathrm{SR}}\right)$. The Fe antiferromagnetic ordering is described by four sublattices. Below $T_{\mathrm{SR}}$ the moments lie almost parallel to the [001] direction with a slight canting giving a small net magnetic moment of $0.15 \mu_{\mathrm{B}}$ along [100] (Lee et al., 2011). Above $T_{\mathrm{SR}}$ the $\mathrm{Fe}$ magnetic moments rotate by $90^{\circ}$, almost parallel to the [100] direction, while the net magnetic moment is now oriented along [001]. Below $5 \mathrm{~K} \mathrm{Sm}^{3+}$ exhibits long-range order with the net magnetic moment antiparallel to the $\mathrm{Fe}$ canted moment (Lee et al., 2011). Recently a ferroelectric polarization was found in this system, which has its origin in the inverted Dzyaloshinskii-Morya interaction (Lee et al., 2011).

We have studied epitaxial thin film of $\mathrm{SmFeO}_{3}(40 \mathrm{~nm}$ thick) grown on $\mathrm{SrTiO}_{3}$ [110] (Locquet et al., 1994). The XMLD measured in cubic systems has been found to be anisotropic (Kunes \& Oppeneer, 2003; Arenholz et al., 2006, 2007; Czekaj et al., 2006; Nolting et al., 2010). Therefore, the study of the spin reorientation in $\mathrm{SmFeO}_{3}$ poses an interesting question for the behavior of the XMLD during the spin reorientation, when the moment does not lie along one of the high-symmetry cubic directions.

We have measured the linear dichroism at the Fe $L_{3,2}$-edges between horizontal and vertical directions as a function of temperature. The measurement was made in normal-incidence geometry, thereby probing the in-plane component of the antiferromagnetic axis. There was no applied magnetic field during the measurements. The result at $2 \mathrm{~K}$ is shown in Fig. 22(a) and is in good agreement with previously published data on single crystals (Joly et al., 2009; Lee et al., 2011). There is a good qualitative agreement between the dichroism measured at $300 \mathrm{~K}$ and an XMLD simulation for cubic $\mathrm{Fe}^{3+}$ published by Arenholz et al. (2006). This indicates that the largest contribution to the linear dichroism measured in

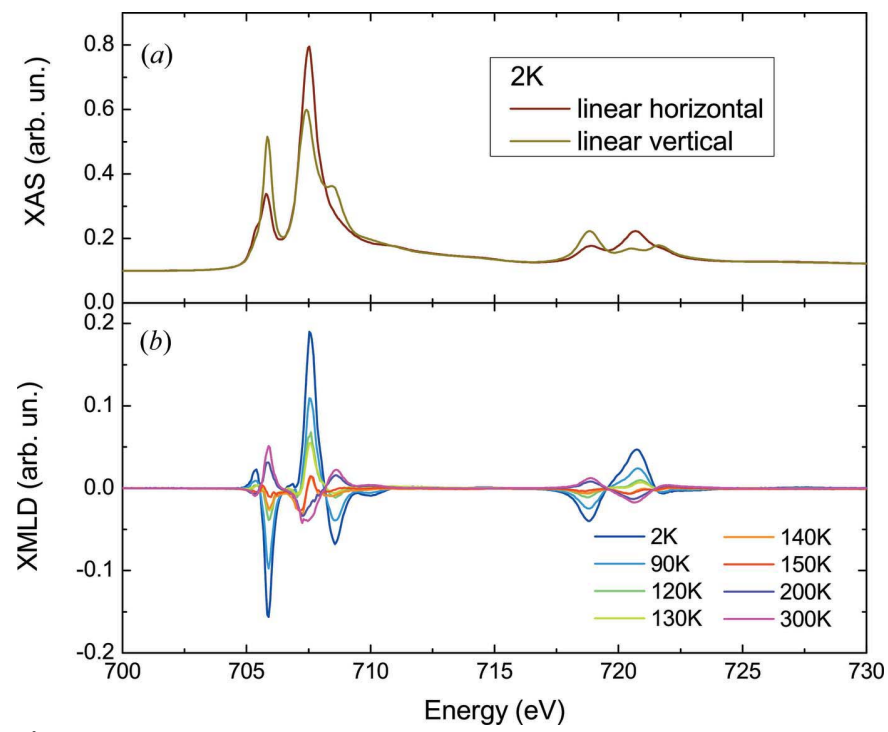

Figure 22

(a) X-ray absorption for linear polarization parallel to the $a$ and $c$ directions in epitaxial $\mathrm{SmFeO}_{3}$ films deposited on $\mathrm{SrTiO}_{3}$ [110] measured at 2 K. (b) XMLD for different sample temperatures.

$\mathrm{SmFeO}_{3}$ is magnetic. It should be pointed out that, strictly speaking, the magnetic dichroism is the difference between the spectra measured by rotating the magnetic moment between parallel and perpendicular to the light polarization. This is not always the same as rotating the light polarization between parallel and perpendicular to the magnetic moment. However, for a cubic or close to cubic system, these two types of dichroism are equivalent (van der Laan et al., 2008).

From the temperature dependence of the linear dichroism shown in Fig. 22(b) one sees an inversion of the linear dichroism between $2 \mathrm{~K}$ and $300 \mathrm{~K}$. This inversion is very likely associated with the reorientation of the Fe spin moments. Even though in bulk systems this reorientation occurs at $480 \mathrm{~K}$ (Lee et al., 2011), it could change in a thin film owing to the strain imposed by the substrate. In the early studies of the spin reorientation of the orthoferrites it was already pointed out that changes in anisotropy or chemical composition can drastically change the spin reorientation temperature (White, 1969). A similar effect was observed in $\mathrm{LaFeO}_{3}$, where a reduction of the Néel temperature between bulk and thin film was measured (Scholl et al., 2000; Seo et al., 2008). This effect was attributed to structural modifications in the unit cell induced by the strain resulting from the lattice mismatch between film and substrate. $\mathrm{SrTiO}_{3}$, used here as a substrate, also presents a structural phase transition, which occurs at $100 \mathrm{~K}$ [varying between 99 and $106 \mathrm{~K}$ depending on the sample (Hünnefeld et al., 2002)], much below the transition we observe in the XMLD data for $\mathrm{SmFeO}_{3}$. Therefore we associate the inversion of the linear dichroism with the $\mathrm{Fe}$ spin reorientation. The intensity of the XMLD is proportional to the magnetic moment squared $\left\langle M^{2}\right\rangle$. This explains the decrease in intensity at $300 \mathrm{~K}$ compared with $2 \mathrm{~K}$ (besides the sign change). Moreover, it could be that the thin film is composed of twinned domains, analogously to what was found previously in $\mathrm{LaFeO}_{3}$ (Lüning et al., 2003; Seo et al., 2008). In 


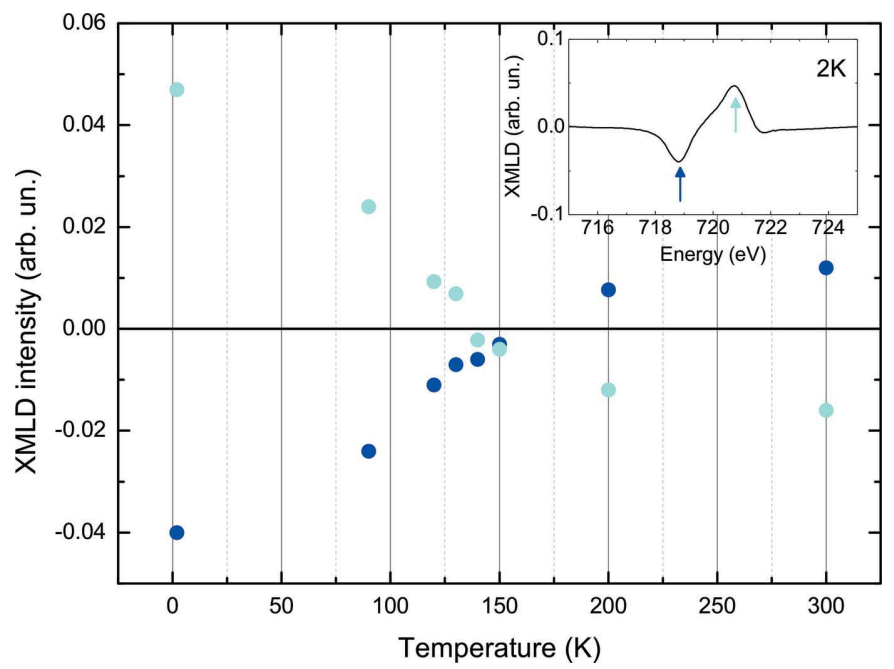

Figure 23

Temperature dependence of the XMLD in $\mathrm{SmFeO}_{3}$. The light and dark blue circles correspond to the maximum and minimum of the linear dichroism at the Fe $L_{2}$ edge. The inset shows the energy position from where these points were taken.

$\mathrm{LaFeO}_{3}$ the domains differ by a rotation in crystallographic orientation by $90^{\circ}$ around $c$. In other words one domain contains $a$ and $c$ in-plane while for the other one $b$ and $c$ are in-plane. If this is also the case for $\mathrm{SmFeO}_{3}$ above the spin reorientation, the linear dichroism gives contrast only for the domain where the $a$ direction is in-plane. Therefore the domain population would also contribute to the weaker dichroic signal at room temperature, compared with $2 \mathrm{~K}$.

Fig. 23 shows the temperature dependence of the maximum and minimum points in the Fe $L_{2}$ XMLD. From this figure it seems that the transition occurs around $150 \mathrm{~K}$.

Fig. 24 shows the linear dichroism as a function of the angle of the incoming light polarization axis $\left(0^{\circ}\right.$ means parallel to

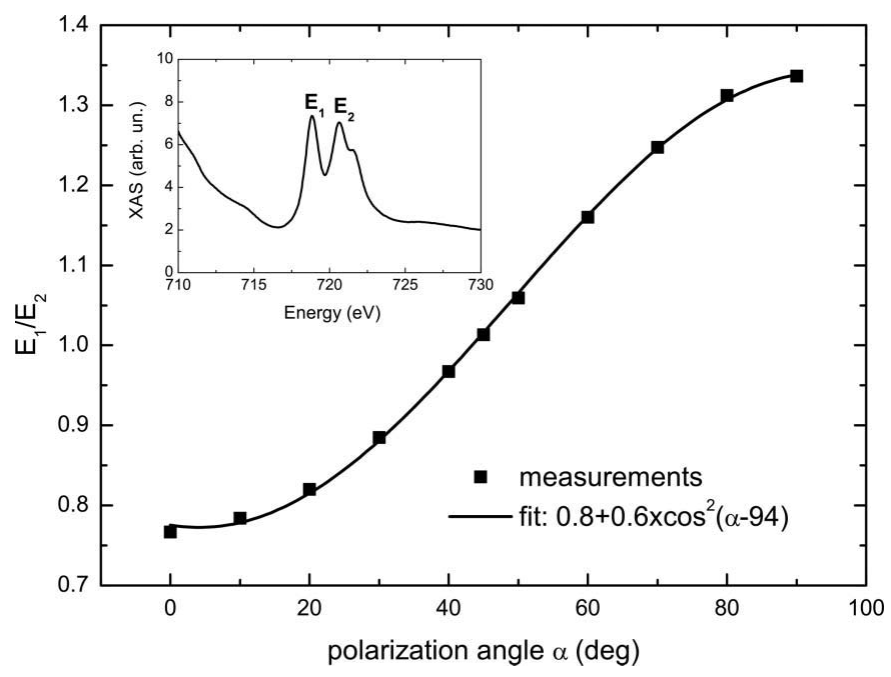

Figure 24

Magnetic linear dichroism as a function of the polarization angle in $\mathrm{SmFeO}_{3}$. The linear dichroism is measured as the ratio between features $E_{1}$ and $E_{2}$ shown in the inset. Measurements were made at $2 \mathrm{~K}$. horizontal plane). Notice that here the XMLD signal is not the difference between two polarization directions as in Fig. 22, since the polarization has been varied during the scan. Instead, the XMLD is taken as the ratio between the two peaks in the $L_{2}$-edge as shown in the inset of Fig. 24. As seen before in Fig. 22(a), these two peaks change their relative weight for the two extreme polarization cases. The data show an $a \cos ^{2} \alpha+b$ dependence. From this dependence and from the shape analysis we conclude that the in-plane component of the spin axis is parallel to the vertical axis.

In summary, we have observed the spin reorientation of $\mathrm{SmFeO}_{3}$ around $150 \mathrm{~K}$ by measuring the temperature dependence of the XMLD at Fe $L$-edges. The spin reorientation seems to take place over a rather wide temperature range, of at least $30 \mathrm{~K}$, but probably wider as the lack of points between $150 \mathrm{~K}$ and $200 \mathrm{~K}$ does not allow us to give the upper temperature bound of the transition region. The measurement of the dependence of the XMLD signal on the polarization angle allows us to find the moment orientation. For the data interpretation during the spin reorientation a more detailed analysis together with multiplet calculations will be carried out.

\section{Concluding remarks}

We have presented the commissioning results of the X-Treme beamline, which operates in the soft X-ray energy range (400$1800 \mathrm{eV}$ ) and is dedicated to polarization-dependent X-ray absorption spectroscopy. The end-station has a $7 \mathrm{~T}-2 \mathrm{~T}$ vector magnet with variable-temperature sample insert enabling sample temperatures between $2 \mathrm{~K}$ and $370 \mathrm{~K}$. The end-station is equipped with an in situ sample preparation system. Two scientific cases showing XMCD and XMLD measurements were presented to exemplify the data quality measured at the beamline.

We acknowledge financial support from the Swiss National Science Foundation (R'Equip program, proposal number 206021-117410) and from the EPFL. We thank Pietro Gambardella for valuable discussions throughout the project. J. W. Seo, J.-P. Locquet and J. Fompeyrine are greatly acknowledged for providing the $\mathrm{SmFeO}_{3}$ sample. We also would like to thank the optics group for their support: Andreas Jaggi, Sibylle Spielmann and Peter Oberta. Blagoj Sarafimov, Juri Honegger and Andrea Steger are acknowledged for the technical support during the beamline installation. Jan Hovind, Dominique Hauenstein and Peter Staeuble are acknowledged for the design of the end-station platform. We are grateful to the EPFL-IPMC workshop for manufacturing parts for the sample preparation system. We would like to mention our great appreciation for the work of the PSI infrastructure groups (water and electrical installation, equipment protection system, survey and alignment, software control, mechanical construction and purchasing), without which this project would not have been possible. We finally thank the PSI management, in particular Friso van der Veen for his enthusiastic support of the present project. 


\section{References}

Arenholz, E., van der Laan, G., Chopdekar, R. V. \& Suzuki, Y. (2006). Phys. Rev. B, 74, 094407.

Arenholz, E., van der Laan, G., Chopdekar, R. V. \& Suzuki, Y. (2007). Phys. Rev. Lett. 98, 197201.

Arrio, M.-A., Scuiller, A., Sainctavit, P., dit Moulin, C. C., Mallah, T. \& Verdaguer, M. (1999). J. Am. Chem. Soc. 121, 6414-6420.

Bahrdt, J., Flechsig, U. \& Senf, F. (1995). Rev. Sci. Instrum. 66, 2719 2723.

Bahrdt, J., Frentrup, W., Gaupp, A., Scheer, M., Gudat, W., Ingold, G. \& Sasaki, S. (2001). Nucl. Instrum. Methods Phys. Res. A, 467-468, 21-29.

Bozorth, R. M., Williams, H. J. \& Walsh, D. E. (1956). Phys. Rev. 103, 572-578.

Carra, P., Thole, B. T., Altarelli, M. \& Wang, X. (1993). Phys. Rev. Lett. 70, 694-697.

Chen, C. T. \& Sette, F. (1989). Rev. Sci. Instrum. 60, 1616-1621.

Chen, C. T., Sette, F., Ma, Y. \& Modest, S. (1990). Phys. Rev. B, 42, 7262-7265.

Chubar, O. \& Elleaume, P. (1998). Proceedings of the Sixth European Particle Accelerator Conference (EPAC'98), p. 1177.

Clarke, J. A. (2004). The Science and Technology of Undulators and Wigglers. Oxford University Press.

Coker, V. S., Gault, A. G., Pearce, C. I., van der Laan, G., Telling, N. D., Charnock, J. M., Polya, D. A. \& Lloyd, J. R. (2006). Environ. Sci. Technol. 40, 7745-7750.

Czekaj, S., Nolting, F., Heyderman, L. J., Willmott, P. R. \& van der Laan, G. (2006). Phys. Rev. B, 73, 020401R.

Dreiser, J., Pedersen, K. S., Piamonteze, C., Rusponi, S., Salman, Z., Ali, M. E., Schau-Magnussen, M., Thuesen, C. A., Piligkos, S., Weihe, H., Mutka, H., Waldmann, O., Oppeneer, P., Bendix, J., Nolting, F. \& Brune, H. (2012). Chem. Sci. 3, 1024-1032.

Erskine, J. L. \& Stern, E. A. (1975). Phys. Rev. B, 12, 5016-5024.

Flechsig, U., Nolting, F., Rodríguez, A. F., Krempaský, J., Quitmann, C., Schmidt, T., Spielmann, S. \& Zimoch, D. (2010). AIP Conf. Proc. 1234, 319-322.

Follath, R. (2001). Nucl. Instrum. Methods Phys. Res. A, 467, 418-425.

Follath, R., Schmidt, J. S., Siewert, F., Holldack, K., Zeschke, T., Frentrup, W., Schmitz, D. \& Sawhney, K. J. S. (2004). AIP Conf. Proc. 705, 348-351.

Follath, R. \& Senf, F. (1997). Nucl. Instrum. Methods Phys. Res. A, 390, 388-394.

Funk, T., Deb, A., George, S. J., Wang, H. \& Cramer, S. P. (2005). Coord. Chem. Rev. 249, 3-30.

Funk, T., Gu, W., Friedrich, S., Wang, H., Gencic, S., Grahame, D. A. \& Cramer, S. P. (2004). J. Am. Chem. Soc. 126, 88-95.

Gambardella, P., Rusponi, S., Veronese, M., Dhesi, S. S., Grazioli, C., Dallmeyer, A., Cabria, I., Zeller, R., Dederichs, P. H., Kern, K., Carbone, C. \& Brune, H. (2003). Science, 300, 1130-1133.

Gullikson, E. M., Korde, R., Canfield, L. R. \& Vest, R. E. (1996). J. Electron Spectrosc. Relat. Phenom. 80, 313-316.

Hünnefeld, T. M., Schneider, J. R., Rutt, U., Rodewald, S., Fleig, J. \& Shirane, G. (2002). Phys. Rev. B, 66, 014113.

Joly, L., Nolting, F., Kimel, A. V., Kirilyuk, A., Pisarev, R. V. \& Rasing, T. (2009). J. Phys. Condens. Matter, 21, 446004.

Kallmayer, M., Schneider, H., Jakob, G., Elmers, H. J., Balke, B. \& Cramm, S. (2007). J. Phys. D, 40, 1552-1560.
Kato, M., Morishita, Y., Oura, M., Yamaoka, H., Tamenori, Y., Okada, K., Matsudo, T., Gejo, T., Suzuki, I. H. \& Saito, N. (2007a). J. Electron Spectrosc. Relat. Phenom. 160, 39-48.

Kato, M., Morishita, Y., Oura, M., Yamaoka, H., Tamenori, Y., Okada, K., Matsudo, T., Gejo, T., Suzuki, I. H. \& Saito, N. (2007b). AIP Conf. Proc. 879, 1121-1124.

Krempaský, J., Flechsig, U., Korhonen, T., Zimoch, D., Quitmann, C. \& Nolting, F. (2010). AIP Conf. Proc. 1234, 705-708.

Kunes, J. \& Oppeneer, P. M. (2003). Phys. Rev. B, 67, 024431.

Laan, G. van der, Arenholz, E., Chopdekar, R. V. \& Suzuki, Y. (2008). Phys. Rev. B, 77, 064407.

Laan, G. van der, Thole, B., Sawatzky, G., Goekoop, J., Fuggle, J., Esteva, J.-M., Karnatak, R., Remeika, J. \& Dabkowska, H. (1986). Phys. Rev. B, 34, 6529-6531.

Lee, J. H., Jeong, Y. K., Park, J. H., Oak, M. A., Jang, H. M., Son, J. Y. \& Scott, J. F. (2011). Phys. Rev. Lett. 107, 117201.

Locquet, J., Catana, A., Mächler, E., Gerber, C. \& Bednorz, J. G. (1994). Appl. Phys. Lett. 64, 372-374.

Lüning, J., Nolting, F., Scholl, A., Ohldag, H., Seo, J. W., Fompeyrine, J., Locquen, J. P. \& Stöhr, J. (2003). Phys. Rev. B, 67, 214433.

Mannini, M., Pineider, F., Sainctavit, P., Danieli, C., Otero, E., Sciancalepore, C., Talarico, A. M., Arrio, M.-A., Cornia, A., Gatteschi, D. \& Sessoli, R. (2009). Nat. Mater. 8, 194-197.

Nolting, F., Legut, D., Rusz, J., Oppeneer, P. M., Woltersdorf, G. \& Back, C. H. (2010). Phys. Rev. B, 82, 184415.

Ohldag, H., Scholl, A., Nolting, F., Arenholz, E., Maat, S., Young, A. T., Carey, M. \& Stöhr, J. (2003). Phys. Rev. Lett. 91, 017203.

Pattrick, R. A. D., van der Laan, G., Henderson, C. M. B., Kuiper, P., Dudzik, E. \& Vaughan, D. J. (2001). Eur. J. Mineral. 14, 1095-1102.

Petersen, H. (1982). Opt. Commun. 40, 402-406.

Petersen, H., Jung, C., Hellwig, C., Peatman, W. B. \& Gudat, W. (1995). Rev. Sci. Instrum. 66, 1-14.

Sasaki, S., Kakuno, K., Takada, T., Shimada, T., Yanagida, K. \& Miyahara, Y. (1993). Nucl. Instrum. Methods Phys. Res. A, 331, $763-767$.

Schaefers, F., Abramsohn, D. \& Krumrey, M. (2002). Reflec, a program to calculate VUV/X-ray optical elements and synchrotron radiation beamline. BESSY, Germany.

Schäfers, F., Mertins, H. C., Gaupp, A., Gudat, W., Mertin, M., Packe, I., Schmolla, F., Di Fonzo, S., Soullié, G., Jark, W., Walker, R., Le Cann, X., Nyholm, R. \& Eriksson, M. (1999). Appl. Opt. 38, 4074 4088.

Scholl, A., Stohr, J., Luning, J., Seo, J. W., Fompeyrine, J., Siegwart, H., Locquet, J., Nolting, F., Anders, S., Fullerton, E. E., Scheinfein, M. R. \& Padmore, H. A. (2000). Science, 287, 1014-1016.

Schütz, G., Wagner, W., Wilhelm, W., Kienle, P., Zeller, R., Frahm, R. \& Materlik, G. (1987). Phys. Rev. Lett. 58, 737-740.

Seo, J. W., Fullerton, E. E., Nolting, F., Scholl, A., Fompeyrine, J. \& Locquet, J.-P. (2008). J. Phys. Condens. Matter, 20, 264014.

Stöhr, J. (1999). J. Magn. Magn. Mater. 200, 470-497.

Stöhr, J. \& König, H. (1995). Phys. Rev. Lett. 75, 3748-3751.

Thole, B. T., Carra, P., Sette, F. \& van der Laan, G. (1992). Phys. Rev. Lett. 68, 1943-1946.

Thole, B. T., van der Laan, G. \& Sawatzky, G. A. (1985). Phys. Rev. Lett. 55, 2086-2088.

Wäckerlin, C., Chylarecka, D., Kleibert, A., Muller, K., Iacovita, C., Nolting, F., Jung, T. A. \& Ballav, N. (2010). Nat. Commun. 1, 61.

White, R. L. (1969). J. Appl. Phys. 40, 1061. 